(n)

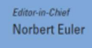
Journal of Nonlinear Mathematical Physics

\title{
Algebro-geometric Constructions of Quasi Periodic Flows of the Discrete Self-dual Network Hierarchy and Applications
}

Dong Gong, Xianguo Geng

To cite this article: Dong Gong, Xianguo Geng (2015) Algebro-geometric Constructions of Quasi Periodic Flows of the Discrete Self-dual Network Hierarchy and Applications, Journal of Nonlinear Mathematical Physics 22:3, 395-427, DOI:

https://doi.org/10.1080/14029251.2015.1079423

To link to this article: https://doi.org/10.1080/14029251.2015.1079423

Published online: 04 January 2021 


\title{
Algebro-geometric Constructions of Quasi Periodic Flows of the Discrete Self-dual Network Hierarchy and Applications
}

\author{
Dong Gong \\ College of Science, Henan Institute of Engineering \\ Zhengzhou, Henan 451191, People's Republic of China \\ gongdong2004@126.com \\ Xianguo Geng* \\ School of Mathematics and Statistics, Zhengzhou University, 100 Kexue Road \\ Zhengzhou, Henan 450001, People's Republic of China \\ xggeng@zzu.edu.cn
}

Received 8 April 2015

Accepted 17 June 2015

\begin{abstract}
In this paper we obtain the discrete integrable self-dual network hierarchy associated with a discrete spectral problem. On the basis of the theory of algebraic curves, the continuous flow and discrete flow related to the discrete self-dual network hierarchy are straightened using the Abel-Jacobi coordinates. The meromorphic function and the Baker-Akhiezer function are introduced on the hyperelliptic curve. Quasi-periodic solutions of the discrete self-dual network hierarchy are constructed with the help of the asymptotic properties and the algebra-geometric characters of the meromorphic function, the Baker-Akhiezer function and the hyperelliptic curve.
\end{abstract}

Keywords: Discrete self-dual network hierarchy; quasi-periodic solutions.

2000 Mathematics Subject Classification: 22E46, 53C35, 57S20

\section{Introduction}

As is well known, in the past few years a lot of work has been done to study the effects of discreteness on the dynamics and physical properties of solitons [1, 16, 18, 20-23]. In these studies, integrable differential difference equations have played an important role since a number of physically interesting problems can be modelled with nonlinear differential-difference equations, including particle vibrations in lattice, currents in electrical networks, pulses in biological chains, etc. Unlike difference equations which are fully discretized, differential-difference equations are semidiscretized with some of their spacial variables discretized while time usually kept continuous. The differential difference equations also play an important part in numerical simulations of nonlinear partial differential equations, queuing problems, and discretizations in solid state and quantum physics. Here we study the nonlinear self-dual network equation

$$
\begin{aligned}
& u_{t}=\left(\varepsilon-u^{2}\right)\left(v-v^{-}\right), \\
& v_{t}=\left(\varepsilon-v^{2}\right)\left(u^{+}-u\right)
\end{aligned}
$$

$\overline{{ }^{*} \text { Corresponding author. }}$ 
associated with the discrete spectral problem

$$
\chi(n+1)=U_{n} \chi(n), \quad U_{n}=\frac{1}{\gamma \pi}\left(\begin{array}{cc}
\lambda+u v & \varepsilon u+\lambda^{-1} v \\
u+\varepsilon \lambda v & \lambda^{-1}+u v
\end{array}\right),
$$

where $u$ and $v$ are two potentials, $\gamma=\sqrt{1-\varepsilon u^{2}}, \quad \pi=\sqrt{1-\varepsilon v^{2}}, \quad \varepsilon= \pm 1$. The nonlinear selfdual network equation describes the propagation of electrical signals in a cascade of four-terminal nonlinear LC self-dual circuits, and was first proposed by Hirota [18], and then studied by Ablowitz and Ladik [1], Göktas and Hereman [16], Shabat and Yamilov [21], Wadati [23], and others [20, 24].

Quasi-periodic (or algebra-geometric, or finite-band) solutions of soliton equations are very important besides of itself interest, which reveal inherent structure mechanism of solutions and describe the quasi-periodic behavior of nonlinear phenomenon or characteristic for the integrability of soliton equations. In a series of literatures, various methods were developed from which quasiperiodic solutions for a lot of soliton equations have been derived, such as the Toda lattice, Kac-van Moerbeke lattice, the relativistic Toda lattice, the discrete $\mathrm{mKdV}$ equation, the discrete nonlinear Schrödinger equation, the discrete Ablowitz-Ladik equation, and so on [2-14, 18].

In this paper, our principal aim is to study straightening out of the discrete self-dual network flows, including the continuous flow and discrete flow, based on the ideas in refs. [12, 14], from which we obtain quasi-periodic solutions of the discrete self-dual network hierarchy. The outline of this paper is as follows. In section 2, we derive the discrete self-dual network hierarchy with the aid of the discrete stationary zero-curvature equation, in which the first nontrivial member is the discrete self-dual network equation. In section 3, we introduce a Lax matrix and establish a direct relation between the elliptic variables and the potentials. The discrete self-dual network hierarchy is separated into solvable ordinary differential equations. In section 4, the hyperelliptic Riemann surface of arithmetic genus $N$ and the Abel-Jacobi coordinates are introduced from which the corresponding continuous flow is straightened out. In the last section, the corresponding discrete flow is straightened out and quasi-periodic solutions of the discrete self-dual network hierarchy are constructed in terms of the Riemann theta functions according to the asymptotic properties and the algebra-geometric characters of the meromorphic function on the hyperelliptic curve.

\section{The hierarchy of discrete self-dual network equations}

In this section, we shall derive the discrete self-dual network hierarchy associated with a discrete spectral problem with two potentials. Throughout this paper we suppose the following hypothesis.

Hypothesis 2.1. Assume that $u$ and $v$ satisfy

$$
\begin{aligned}
& u(\cdot, t), v(\cdot, t) \in \mathbb{C}^{\mathbb{Z}}, \quad t \in \mathbb{R}, \quad u(n, \cdot), v(n, \cdot) \in C^{1}(\mathbb{R}), \quad n \in \mathbb{Z}, \\
& u(n, t) v(n, t) \neq 0, \quad(n, t) \in \mathbb{Z} \times \mathbb{R},
\end{aligned}
$$

where $u=u(n, t), v=v(n, t)$, and $\mathbb{C}^{\mathbb{Z}}$ denotes the set of all complex-valued sequences indexed by $\mathbb{Z}$. For the sake of convenience, we denote by $E^{ \pm}$the shift operators acting on complex-valued sequences $f=\{f(n)\}_{n \in \mathbb{Z}}$ according to

$$
\left(E^{ \pm} f\right)(n)=f(n \pm 1), \quad n \in \mathbb{Z} .
$$

and define difference operator $\triangle=E-1$. Moreover, we will frequently use the notation

$$
f^{ \pm}=E^{ \pm} f, \quad f \in \mathbb{C}^{\mathbb{Z}}
$$


Let us consider the discrete spectral problem

$$
\chi(n+1)=U_{n} \chi(n), \quad U_{n}=\frac{1}{\gamma \pi}\left(\begin{array}{cc}
\lambda+u v & \varepsilon u+\lambda^{-1} v \\
u+\lambda \varepsilon v & \lambda^{-1}+u v
\end{array}\right),
$$

where $u, v$ are two potentials, $\lambda$ is a constant spectral parameter,

$$
\gamma=\sqrt{1-\varepsilon u^{2}}, \quad \pi=\sqrt{1-\varepsilon v^{2}}, \quad \varepsilon= \pm 1 .
$$

Proposition 2.2. Let two matrices $V_{n}$ and $\hat{V}_{n}$ satisfy

$$
\hat{V}_{n} U_{n}^{(1)}-U_{n}^{(1)} V_{n}=0, \quad V_{n}^{+} U_{n}^{(2)}-U_{n}^{(2)} \hat{V}_{n}=0,
$$

where $\left(z^{2}=\lambda\right)$

$$
U_{n}^{(1)}=\frac{1}{\gamma}\left(\begin{array}{cc}
z & z^{-1} \varepsilon u \\
z u & z^{-1}
\end{array}\right), \quad U_{n}^{(2)}=\frac{1}{\pi}\left(\begin{array}{cc}
z & z^{-1} v \\
z \varepsilon v & z^{-1}
\end{array}\right) .
$$

Then $V_{n}$ satisfies the discrete stationary zero-curvature equation

$$
V_{n}^{+} U_{n}-U_{n} V_{n}=0,
$$

and $\operatorname{det} V_{n}$ and $\operatorname{det} \hat{V}_{n}$ are constants independent of $\mathrm{n}$.

Proof. Noticing $U_{n}=U_{n}^{(2)} U_{n}^{(1)}$, we have

$$
V_{n}^{+} U_{n}-U_{n} V_{n}=\left(V_{n}^{+} U_{n}^{(2)}-U_{n}^{(2)} \hat{V}_{n}\right) U_{n}^{(1)}+U_{n}^{(2)}\left(\hat{V}_{n} U_{n}^{(1)}-U_{n}^{(1)} V_{n}\right)=0 .
$$

By(2.2), we arrive at $\operatorname{det} V_{n}^{+}=\operatorname{det} \hat{V}_{n}$ and $\operatorname{det} \hat{V}_{n}=\operatorname{det} V_{n}$, which imply that $\operatorname{det} V_{n}$ and $\operatorname{det} \hat{V}_{n}$ are constants independent of $n$.

Assume that solutions $V_{n}$ and $\hat{V}_{n}$ of (2.2) take the form

$$
V_{n}=\left(\begin{array}{cc}
\widehat{G} & \widehat{F} \\
\lambda \widehat{H} & -\widehat{G}
\end{array}\right), \quad \hat{V}_{n}=\left(\begin{array}{cc}
\hat{a} & \hat{b} \\
\lambda \hat{c} & -\hat{a}
\end{array}\right) .
$$

Then (2.2) can be written as

$$
\begin{aligned}
& \hat{a}-\widehat{G}+u \hat{b}-\varepsilon u \widehat{H}=0, \\
& \hat{b}-\lambda \widehat{F}+\varepsilon u(\hat{a}+\widehat{G})=0, \\
& \lambda \hat{c}-\widehat{H}-u(\hat{a}+\widehat{G})=0, \\
& \widehat{G}-\hat{a}+\lambda \varepsilon u \hat{c}-\lambda u \widehat{F}=0, \\
& \\
& \widehat{G}^{+}-\hat{a}+\varepsilon v \widehat{F}^{+}-v \hat{c}=0, \\
& \widehat{F}^{+}-\lambda \hat{b}+v\left(\hat{a}+\widehat{G}^{+}\right)=0, \\
& \lambda \widehat{H}^{+}-\hat{c}-\varepsilon v\left(\hat{a}+\widehat{G}^{+}\right)=0, \\
& \hat{a}-\widehat{G}^{+}-\varepsilon \lambda v \hat{b}+\lambda v \widehat{H}^{+}=0 .
\end{aligned}
$$

Let

$$
\widehat{G}=\sum_{j=0}^{\infty} \hat{g}_{j,+} \lambda^{-j}, \quad \widehat{F}=\sum_{j=0}^{\infty} \hat{f}_{j,+} \lambda^{-j}, \quad \widehat{H}=\sum_{j=0}^{\infty} \hat{h}_{j,+} \lambda^{-j}
$$




$$
\hat{a}=\sum_{j=0}^{\infty} \hat{a}_{j,+} \lambda^{-j}, \quad \hat{b}=\sum_{j=0}^{\infty} \hat{b}_{j,+} \lambda^{-j}, \quad \hat{c}=\sum_{j=0}^{\infty} \hat{c}_{j,+} \lambda^{-j}
$$

with the condition

$$
\widehat{G}^{2}+\lambda \widehat{F} \widehat{H}=\frac{1}{4}, \quad \hat{a}^{2}+\lambda \hat{b} \hat{c}=\frac{1}{4} .
$$

Substituting (2.8) into (2.6), (2.7) and (2.9) and comparing the coefficients of the same power for $\lambda$, we obtain

$$
\begin{gathered}
\hat{g}_{0,+}=\frac{1}{2}, \quad \hat{f}_{0,+}=0, \quad \hat{h}_{0,+}=0, \\
\hat{a}_{0,+}=\frac{1}{2}, \quad \hat{b}_{0,+}=0, \quad \hat{c}_{0,+}=0, \\
\hat{a}_{j,+}-\hat{g}_{j,+}+u \hat{b}_{j,+}-\varepsilon u \hat{h}_{j,+}=0, \\
\hat{b}_{j,+}-\hat{f}_{j+1,+}+\varepsilon u\left(\hat{a}_{j,+}+\hat{g}_{j,+}\right)=0, \\
\hat{c}_{j+1,+}-\hat{h}_{j,+}-u\left(\hat{a}_{j,+}+\hat{g}_{j,+}=0,\right. \\
\hat{g}_{j,+}-\hat{a}_{j,+}+\varepsilon u \hat{c}_{j+1,+}-u \hat{f}_{j+1,+}=0, \\
\hat{g}_{j,+}^{+}-\hat{a}_{j,+}+\varepsilon v \hat{f}_{j,+}^{+}-v \hat{c}_{j,+}=0, \\
\hat{f}_{j,+}^{+}-\hat{b}_{j+1,+}+v\left(a_{j,+}+\hat{g}_{j,+}^{+}\right)=0, \\
\hat{h}_{j+1,+}^{+}-\hat{c}_{j,+}-\varepsilon v\left(\hat{a}_{j,+}+\hat{g}_{j,+}^{+}\right)=0, \\
\hat{a}_{j,+}-\hat{g}_{j,+}^{+}-\varepsilon v \hat{b}_{j+1,+}+v \hat{h}_{j,+}^{+}=0, \\
\sum_{k=0}^{j} \hat{g}_{k,+} \hat{g}_{j-k,+}+\sum_{k=0}^{j+1} \hat{f}_{k,+} \hat{h}_{j-k+1,+}=0, \\
\sum_{k=0}^{j} \hat{a}_{k,+} \hat{a}_{j-k,+}+\sum_{k=0}^{j+1} \hat{b}_{k,+} \hat{c}_{j-k+1,+}=0 \quad(j \geq 1) .
\end{gathered}
$$

Then $\hat{g}_{j,+}, \hat{f}_{j,+}, \hat{h}_{j,+}, \hat{a}_{j,+}, \hat{b}_{j,+}, \hat{c}_{j,+}$ are uniquely determined by the recursion relations (2.10)-(2.13). It is easy to see that

$$
\begin{aligned}
& \hat{g}_{1,+}=-u v^{-}, \quad \hat{f}_{1,+}=\varepsilon u, \quad \hat{h}_{1,+}=\varepsilon v^{-}, \\
& \hat{g}_{2,+}=-\varepsilon\left(u u^{-}+v v^{-}\right)+u v^{-}\left(u v+u v^{-}+u^{-} v^{-}\right), \\
& \hat{f}_{2,+}=v-\varepsilon u\left(u v^{-}+u v\right), \quad \hat{h}_{2,+}=u^{-}-2 \varepsilon v^{-}\left(u v^{-}+u^{-} v^{-}\right), \\
& \hat{a}_{1,+}=-u v, \quad \hat{b}_{1,+}=v, \quad \hat{c}_{1,+}=u, \\
& \hat{a}_{2,+}=-\varepsilon\left(u u^{+}+v v^{-}\right)+u v\left(u^{+} v+u v+u v^{-}\right), \\
& \hat{b}_{2,+}=\varepsilon u^{+}-v\left(u^{+} v+u v\right), \quad \hat{c}_{2,+}=\varepsilon v^{-}-u\left(u v^{-}+u v\right) .
\end{aligned}
$$

On the other hand, we consider the following assumption:

$$
\begin{array}{ccc}
\widehat{G}=\sum_{j=0}^{\infty} \hat{g}_{j,-} \lambda^{j}, & \widehat{F}=\sum_{j=0}^{\infty} \hat{f}_{j,-} \lambda^{j}, & \widehat{H}=\sum_{j=0}^{\infty} \hat{h}_{j,-} \lambda^{j}, \\
\hat{a}=\sum_{j=0}^{\infty} \hat{a}_{j,-} \lambda^{j}, & \hat{b}=\sum_{j=0}^{\infty} \hat{b}_{j,-} \lambda^{j}, & \hat{c}=\sum_{j=0}^{\infty} \hat{c}_{j,-} \lambda^{j}
\end{array}
$$


with the condition

$$
\widehat{G}^{2}+\lambda \widehat{F} \widehat{H}=\frac{1}{4}, \quad \hat{a}^{2}+\lambda \hat{b} \hat{c}=\frac{1}{4} .
$$

Substituting (2.15) into (2.6), (2.7) and (2.16), we obtain

$$
\begin{gathered}
\hat{g}_{0,-}=-\frac{1}{2}, \quad \hat{f}_{0,-}=v^{-}, \quad \hat{h}_{0,-}=u, \\
\hat{a}_{0,-}=-\frac{1}{2}, \quad \hat{b}_{0,-}=\varepsilon u, \quad \hat{c}_{0,-}=\varepsilon v, \\
\hat{a}_{j,-}-\hat{g}_{j,-}+u \hat{b}_{j,-}-\varepsilon u \hat{h}_{j,-}=0, \\
\hat{b}_{j,-}-\hat{f}_{j-1,-}+\varepsilon u\left(\hat{a}_{j,-}+\hat{g}_{j,-}\right)=0, \\
\hat{c}_{j-1,-}-\hat{h}_{j,-}-u\left(\hat{a}_{j,-}+\hat{g}_{j,-}=0,\right. \\
\hat{g}_{j,-}-\hat{a}_{j,-}+\varepsilon u \hat{c}_{j-1,-}-u \hat{f}_{j-1,-}=0, \\
\hat{g}_{j,-}^{+}-\hat{a}_{j,-}+\varepsilon v \hat{f}_{j,-}^{+}-v \hat{c}_{j,-}=0, \\
\hat{f}_{j,-}^{+}-\hat{b}_{j-1,-}+v\left(\hat{a}_{j,-}+\hat{g}_{j,-}^{+}\right)=0, \\
\hat{h}_{j-1,-}^{+}-\hat{c}_{j,-}-\varepsilon v\left(\hat{a}_{j,-}+\hat{g}_{j,-}^{+}\right)=0, \\
\hat{a}_{j,-}-\hat{g}_{j,-}^{+}-\varepsilon v \hat{b}_{j-1,-}+v \hat{h}_{j-1,-}^{+}=0, \\
\sum_{k=0}^{j} \hat{g}_{k,-} \hat{g}_{j-k,-}+\sum_{k=0}^{j-1} \hat{f}_{k,-} \hat{h}_{j-k-1,-}=0, \\
\sum_{k=0}^{j} \hat{a}_{k,-} \hat{a}_{j-k,-}+\sum_{k=0}^{j-1} \hat{b}_{k,-} \hat{c}_{j-k-1,-}=0 \quad(j \geq 1) .
\end{gathered}
$$

Then $\hat{g}_{j,-}, \hat{f}_{j,-}, \hat{h}_{j,-}, \hat{a}_{j,-}, \hat{b}_{j,-}, \hat{c}_{j,-}$ are uniquely determined by the recursion relations (2.17)-(2.20), and

$$
\begin{aligned}
& \hat{g}_{1,-}=u v^{-}, \quad \hat{f}_{1,-}=\varepsilon u^{-}-v^{-}\left(u v^{-}+u^{-} v^{-}\right) \\
& \hat{h}_{1,-}=\varepsilon v-u\left(u v^{-}+u v\right) \\
& \hat{g}_{2,-}=\varepsilon\left(u u^{-}+v v^{-}\right)-u v^{-}\left(u v+u v^{-}+u^{-} v^{-}\right), \\
& \hat{a}_{1,-}=u v, \quad \hat{b}_{1,-}=v^{-}-\varepsilon u\left(u v^{-}+u v\right) \\
& \hat{c}_{1,-}=u^{+}-\varepsilon v\left(u^{+} v+u v\right) \\
& \hat{a}_{2,-}=\varepsilon\left(u u^{+}+v v^{-}\right)-u v\left(u^{+} v+u v+u v^{-}\right) .
\end{aligned}
$$

Proposition 2.3. Let two matrices $V_{n}^{(\underline{m})}$ and $\hat{V}_{n}{ }^{(\underline{m})}$ be solutions of equations

$$
U_{n t_{\underline{m}}}^{(1)}=\hat{V}_{n}^{(\underline{m})} U_{n}^{(1)}-U_{n}^{(1)} V_{n}^{(\underline{m})}, \quad U_{n t_{\underline{m}}}^{(2)}=V_{n+1}^{(\underline{m})} U_{n}^{(2)}-U_{n}^{(2)} \hat{V}_{n}^{(\underline{m})},
$$

which imply that $V_{n}^{(\underline{m})}$ satisfies the discrete zero-curvature equation

$$
U_{n t_{\underline{m}}}=V_{n+1}^{(\underline{m})} U_{n}-U_{n} V_{n}^{(\underline{m})},
$$

where $U_{n}^{(1)}$ and $U_{n}^{(2)}$ are defined by (2.3).

Proof. By using (2.2), a direct calculation shows that

$$
\begin{aligned}
U_{n t_{\underline{m}}}-V_{n+1}^{(\underline{m})} U_{n}+U_{n} V_{n}^{(\underline{\underline{m}})}= & \left(U_{n t_{m}}^{(2)}-V_{n+1}^{(\underline{m})} U_{n}^{(2)}+U_{n}^{(2)} \hat{V}_{n}^{(\underline{m})}\right) U_{n}^{(1)} \\
& +U_{n}^{(2)}\left(U_{n t_{\underline{m}}}^{(1)}-\hat{V}_{n}^{(\underline{m})} U_{n}^{(1)}+U_{n}^{(1)} V_{n}^{(\underline{m})}\right)=0 .
\end{aligned}
$$


Let $V_{n}^{(\underline{m})}$ and $\hat{V}_{n}^{(\underline{m})}$ of (2.22) take the form

$$
V_{n}^{(\underline{m})}=\left(\begin{array}{cc}
\widetilde{G}_{\underline{m}} & \widetilde{F}_{\underline{m}} \\
\lambda \widetilde{\widetilde{H}}_{\underline{m}} & -\widetilde{\widetilde{G}}_{\underline{m}}
\end{array}\right), \quad \hat{V}_{n}^{(\underline{m})}=\left(\begin{array}{cc}
\tilde{a}_{\underline{m}} & \tilde{b}_{\underline{m}} \\
\lambda \tilde{\tilde{c}}_{\underline{\underline{m}}} & -\tilde{\tilde{a}}_{\underline{m}}
\end{array}\right), \quad \underline{m}=(m, m) \in \mathbb{N}_{0}^{2},
$$

where $\widetilde{G}_{\underline{m}}, \widetilde{F}_{\underline{m}}, \widetilde{H}_{\underline{m}}, \tilde{a}_{\underline{m}}, \tilde{b}_{\underline{m}}, \tilde{c}_{\underline{m}}$ are polynomials of the spectral parameter $\lambda$, which take the form

$$
\begin{aligned}
& \widetilde{G}_{\underline{m}}=\sum_{j=0}^{m} \tilde{g}_{j,+} \lambda^{m-j}+\sum_{j=0}^{m} \tilde{g}_{j,-} \lambda^{j-m}, \\
& \widetilde{F}_{\underline{m}}=\sum_{j=0}^{m} \tilde{f}_{j,+} \lambda^{m-j}+\sum_{j=0}^{m-1} \tilde{f}_{j,-} \lambda^{j-m}, \\
& \widetilde{H}_{\underline{m}}=\sum_{j=0}^{m} \tilde{h}_{j,+} \lambda^{m-j}+\sum_{j=0}^{m-1} \tilde{h}_{j,-} \lambda^{j-m}, \\
& \tilde{a}_{\underline{m}}=\sum_{j=0}^{m} \tilde{a}_{j,+} \lambda^{m-j}+\sum_{j=0}^{m} \tilde{a}_{j,-} \lambda^{j-m}, \\
& \tilde{b}_{\underline{m}}=\sum_{j=0}^{m} \tilde{b}_{j,+} \lambda^{m-j}+\sum_{j=0}^{m-1} \tilde{b}_{j,-} \lambda^{j-m}, \\
& \tilde{c}_{\underline{m}}=\sum_{j=0}^{m} \tilde{c}_{j,+} \lambda^{m-j}+\sum_{j=0}^{m-1} \tilde{c}_{j,-} \lambda^{j-m} .
\end{aligned}
$$

with $\tilde{g}_{j, \pm}=\sum_{s=0}^{j} \tilde{\alpha}_{j-s,+} \hat{g}_{j, \pm}, \quad \tilde{f}_{j, \pm}=\sum_{s=0}^{j} \tilde{\alpha}_{j-s,+} \hat{f}_{j, \pm}, \quad \tilde{h}_{j, \pm}=\sum_{s=0}^{j} \tilde{\alpha}_{j-s,+} \hat{h}_{j, \pm}, \quad \tilde{a}_{j, \pm}=\sum_{s=0}^{j} \tilde{\alpha}_{j-s,+} \hat{a}_{j, \pm}$, $\tilde{b}_{j, \pm}=\sum_{s=0}^{j} \tilde{\alpha}_{j-s,+} \hat{b}_{j, \pm}, \quad \tilde{c}_{j, \pm}=\sum_{s=0}^{j} \tilde{\alpha}_{j-s,+} \hat{c}_{j, \pm}, \tilde{\alpha}_{j,+} \quad(0 \leq j \leq m)$ are constants. Then (2.22) can be written as

$$
\begin{aligned}
& \gamma\left(\gamma^{-1}\right)_{t_{\underline{m}}}=\tilde{a}_{\underline{m}}-\widetilde{G}_{\underline{m}}+u \tilde{b}_{\underline{m}}-\varepsilon u \widetilde{H}_{\underline{m}}, \\
& \varepsilon u_{t_{\underline{m}}}+\varepsilon u \gamma\left(\gamma^{-1}\right)_{t_{\underline{m}}}=\tilde{b}_{\underline{m}}-\lambda \widetilde{F}_{\underline{m}}+\varepsilon u\left(\tilde{a}_{\underline{m}}+\widetilde{G}_{\underline{m}}\right), \\
& u_{t_{\underline{m}}}+u \gamma\left(\gamma^{-1}\right)_{t_{\underline{m}}}=\lambda \tilde{c}_{\underline{m}}-\widetilde{H}_{\underline{m}}-u\left(\tilde{a}_{\underline{m}}+\widetilde{G}_{\underline{m}}\right), \\
& \gamma\left(\gamma^{-1}\right)_{t_{\underline{m}}}=\tilde{G}_{\underline{m}}^{\underline{m}}-\tilde{a}_{\underline{m}}+\lambda \varepsilon u \tilde{c}_{\underline{m}}-\lambda u \widetilde{F}_{m}, \\
& \pi\left(\pi^{-1}\right)_{t_{\underline{m}}}=\widetilde{G}_{\underline{m}}^{+}-\tilde{a}_{\underline{m}}+\varepsilon v \widetilde{F}_{m}^{+}-v \tilde{c}_{\underline{m}}, \\
& v_{t_{\underline{m}}}+v \pi\left(\pi^{-1}\right)_{t_{\underline{m}}}=\widetilde{F}_{\underline{m}}^{+}-\lambda \tilde{b}_{\underline{m}}+v\left(\widetilde{a}_{\underline{m}}+\widetilde{G}_{\underline{m}}^{+}\right), \\
& \varepsilon v_{t_{\underline{m}}}+\varepsilon v \pi\left(\pi^{-1}\right)_{t_{\underline{m}}}=\lambda \widetilde{H}_{\underline{m}}^{+}-\widetilde{c}_{\underline{m}}-\varepsilon v\left(\tilde{a}_{\underline{m}}+\widetilde{G}_{\underline{m}}^{+}\right), \\
& \pi\left(\pi^{-1}\right)_{t_{\underline{m}}}=\tilde{a}_{\underline{m}}-\widetilde{G}_{\underline{m}}^{+}-\lambda \varepsilon v \tilde{b}_{\underline{m}}+\lambda v \widetilde{H}_{\underline{m}}^{+},
\end{aligned}
$$

that is

$$
\begin{aligned}
& \left(1-\varepsilon u^{2}\right)\left(\varepsilon \tilde{b}_{m,+}-\tilde{c}_{m-1,-}\right)+\left(1+\varepsilon u^{2}\right)\left(\tilde{h}_{m,+}-\varepsilon \tilde{f}_{m-1,-}\right)+4 u\left(\tilde{g}_{m,+}+\tilde{g}_{m,-}\right)=0, \\
& \left(1-\varepsilon v^{2}\right)\left(\tilde{f}_{m}^{+}-\varepsilon \tilde{h}_{m-1,-}^{+}\right)+\left(1+\varepsilon v^{2}\right)\left(\varepsilon \tilde{c}_{m,+}-\tilde{b}_{m-1,-}\right)+4 v\left(\tilde{a}_{m,+}+\tilde{a}_{m,-}\right)=0,
\end{aligned}
$$

and

$$
\begin{aligned}
& u_{t_{\underline{m}}}=\frac{1}{2}\left(1-\varepsilon u^{2}\right)\left(\varepsilon \tilde{b}_{m,+}-\tilde{h}_{m,+}-\varepsilon \tilde{f}_{m-1,-}+\tilde{c}_{m-1,-}\right), \\
& v_{\underline{\underline{m}}}=\frac{1}{2}\left(1-\varepsilon v^{2}\right)\left(\tilde{f}_{m,+}^{+}-\varepsilon \tilde{c}_{m,+}-\tilde{b}_{m-1,-}+\varepsilon \tilde{h}_{m-1,+}\right), \quad \underline{m}=(m, m) \in \mathbb{N}_{0}^{2} .
\end{aligned}
$$

Iterating (2.11)-(2.13) and (2.18)-(2.20), a direct calculation shows that (2.28) is an identity. It is just the discrete self-dual network hierarchy for varying $\underline{m}=(m, m) \in \mathbb{N}_{0}^{2}$ in Eq. (2.29), and the first 
nontrivial equation in this hierarchy is the discrete self-dual network equation

$$
\begin{aligned}
& u_{t}=\left(\varepsilon-u^{2}\right)\left(v-v^{-}\right), \\
& v_{t}=\left(\varepsilon-v^{2}\right)\left(u^{+}-u\right) .
\end{aligned}
$$

for $\widetilde{\alpha}_{0,+}=\widetilde{\alpha}_{0,-}=1, \quad \underline{m}=(1,1), \quad t_{(1,1)}=t$.

\section{Evolution of the elliptic variables}

In what follows, we will establish a relation between the elliptic variables and the potentials. Let $\psi(n)=\left(\psi_{1}(n), \psi_{2}(n)\right)^{\mathrm{T}}$ and $\varphi(n)=\left(\varphi_{1}(n), \varphi_{2}(n)\right)^{\mathrm{T}}$ be two basic solutions of (2.1) and (2.23). We introduce a Lax matrix

$$
W_{n}=\frac{1}{2}\left(\psi(n) \varphi(n)^{\mathrm{T}}+\varphi(n) \psi(n)^{\mathrm{T}}\right)\left(\begin{array}{cc}
0 & -1 \\
1 & 0
\end{array}\right)=\left(\begin{array}{cc}
G(n) & F(n) \\
\lambda H(n) & -G(n)
\end{array}\right),
$$

which satisfies the Lax equations

$$
W_{n+1} U_{n}-U_{n} W_{n}=0, \quad W_{n, \underline{\underline{m}}}=\left[V_{n}^{(\underline{m})}, W_{n}\right] .
$$

Therefore, $\operatorname{det} W$ is a constant independent of $n$ and $t_{\underline{m}}$. In fact, we obtain by the first expression of (3.2) that $W_{n+1}=U_{n} W_{n} U_{n}^{-1}$. Then $\operatorname{det} W_{n+1}=\operatorname{det} W_{n}$, which means that $\operatorname{det} W_{n}$ is independent of $n$. In a way similar to the continuous case, a direct calculation shows that $\left(\operatorname{det} W_{n}\right)_{t_{\underline{m}}}=0$. Equation (3.2) can be written as

$$
\begin{aligned}
& (\lambda+u v) \triangle G+(u+\lambda \varepsilon v) F^{+}-(\lambda \varepsilon u+v) H=0, \\
& (\varepsilon u+\lambda-1 v)\left(G^{+}+G\right)+(\lambda-1+u v) F^{+}-(\lambda+u v) F=0, \\
& (u+\lambda \varepsilon v)\left(G^{+}+G\right)-\lambda(\lambda+u v) H^{+}+(1+\lambda u v) H=0, \\
& \left(\lambda^{-1}+u v\right) \triangle G-(\lambda \varepsilon u+v) H^{+}+(u+\lambda \varepsilon v) F=0,
\end{aligned}
$$

and

$$
\begin{aligned}
& G(n)_{t_{\underline{m}}}=\lambda \widetilde{F}_{\underline{m}}(n) H(n)-\lambda \widetilde{H}_{\underline{m}}(n) F(n), \\
& F(n)_{t_{\underline{m}}}=2 \widetilde{G}_{\underline{m}}(n) F(n)-2 \widetilde{F}_{\underline{m}}(n) G(n), \\
& H(n)_{t_{\underline{m}}}=2 \widetilde{H}_{\underline{m}}(n) G(n)-2 \widetilde{G}_{\underline{m}}(n) H(n) .
\end{aligned}
$$

Now we suppose the functions $G(n), F(n)$ and $H(n)$ are finite-order polynomials in $\lambda$ :

$$
\begin{aligned}
& G(n)=\sum_{j=0}^{N_{1}} g_{j,+}(n) \lambda^{N_{1}-j}+\sum_{j=0}^{N_{2}-1} g_{j,-}(n) \lambda^{-N_{2}+j}, \\
& F(n)=\sum_{j=0}^{N_{1}} f_{j,+}(n) \lambda^{N_{1}-j}+\sum_{j=0}^{N_{2}-1} f_{j,-}(n) \lambda^{-N_{2}+j}, \\
& H(n)=\sum_{j=0}^{N_{1}} h_{j,+}(n) \lambda^{N_{1}-j}+\sum_{j=0}^{N_{2}-1} h_{j,-}(n) \lambda^{-N_{2}+j}
\end{aligned}
$$


Substituting (3.5) into (3.3) yields

$$
\begin{aligned}
& g_{0,+}=\frac{1}{2} \alpha_{0,+}, \quad f_{0,+}=0, \quad h_{0,+}=0, \\
& g_{1,+}=-\alpha_{0,+} u v^{-}+\frac{1}{2} \alpha_{1,+}, \quad f_{1,+}=\varepsilon \alpha_{0,+} u, \quad h_{1,+}=\varepsilon \alpha_{0,+} v^{-}, \\
& g_{2,+}=-\varepsilon \alpha_{0+}\left(u u^{-}+v v^{-}\right)+\alpha_{0,+} u v^{-}\left(u v+u v^{-}+u^{-} v^{-}\right)-\alpha_{1,+} u v^{-}+\frac{1}{2} \alpha_{2,+}, \\
& f_{2,+}=\alpha_{0+} v-\varepsilon \alpha_{0+} u\left(u v^{-}+u v\right)+\varepsilon \alpha_{1,+} u, \\
& h_{2,+}=\alpha_{0+} u^{-}-\varepsilon \alpha_{0+} v^{-}\left(u v^{-}+u^{-} v^{-}\right)+\varepsilon \alpha_{1,+} v^{-}, \\
& g_{0,-}=-\frac{1}{2} \alpha_{0,-}, \quad f_{0,-}=\alpha_{0,-} v^{-}, \quad h_{0,-}=\alpha_{0,-} u, \\
& g_{1,-}=\alpha_{0,-} u v^{-}-\frac{1}{2} \alpha_{1,-}, \quad f_{1,-}=\varepsilon \alpha_{0,-} u^{-}-\alpha_{0,-} v^{-}\left(u v^{-}+u^{-} v^{-}\right)+\alpha_{1,-} v^{-}, \\
& h_{1,-}=\varepsilon \alpha_{0,-} v-\alpha_{0,-} u\left(u v^{-}+u v\right)+\alpha_{1,-} u, \\
& g_{2,-}=\varepsilon \alpha_{0,-}\left(u u^{-}+v v^{-}\right)-\alpha_{0,-} u v^{-}\left(u v+u v^{-}+u^{-} v^{-}\right)+\alpha_{1,-} u v^{-}-\frac{1}{2} \alpha_{2,-} .
\end{aligned}
$$

Subsequently, it will be useful to work with the homogeneous coefficients, $\hat{g}_{j, \pm}, \hat{f}_{j, \pm}$, and $\hat{h}_{j, \pm}$, defined by the vanishing of all summation constants $\alpha_{k, \pm}$ for $k=1,2, \ldots, j$, and $\alpha_{0, \pm}=1$,

$$
\begin{aligned}
& \hat{g}_{0,+}(n)=\frac{1}{2}, \quad \hat{g}_{0,-}(n)=-\frac{1}{2}, \quad \hat{g}_{j}(n)=\left.g_{j}(n)\right|_{\alpha_{0, \pm}=1, \alpha_{k, \pm}=0, k=1, \ldots, j}, \quad j \in \mathbb{N}, \\
& \hat{f}_{0,+}(n)=0, \quad \hat{f}_{0,-}(n)=v^{-}, \quad \hat{f}_{j}(n)=\left.f_{j}(n)\right|_{\alpha_{0, \pm}=1, \alpha_{k, \pm}=0, k=1, \ldots, j}, \quad j \in \mathbb{N}, \\
& \hat{h}_{0,+}(n)=0, \quad \hat{h}_{0,-}(n)=u, \quad \hat{h}_{j}(n)=\left.h_{j}(n)\right|_{\alpha_{0, \pm}=1, \alpha_{k, \pm}=0, k=1, \ldots, j}, \quad j \in \mathbb{N} .
\end{aligned}
$$

By induction one infers that

$$
g_{j, \pm}(n)=\sum_{l=0}^{k} \alpha_{j-l, \pm} \hat{g}_{k, \pm}(n), \quad f_{j, \pm}(n)=\sum_{l=0}^{k} \alpha_{j-l, \pm} \hat{f}_{k, \pm}(n), \quad h_{j, \pm}(n)=\sum_{l=0}^{k} \alpha_{j-l, \pm} \hat{h}_{k, \pm}(n) .
$$

Similarly, the corresponding homogeneous polynomials are defined by $(l \in \mathbb{N})$

$$
\begin{aligned}
& \widehat{F}_{0, \pm}=0, \quad \widehat{F}_{l,+}(\lambda)=\sum_{k=0}^{l-1} \hat{f}_{l-k,+}(n) \lambda^{k}, \quad \widehat{F}_{l,-}(\lambda)=\sum_{k=1}^{l} \hat{f}_{l-k,-}(n) \lambda^{-k}, \\
& \widehat{G}_{0,+}=\frac{1}{2}, \quad \widehat{G}_{l,+}(\lambda)=\sum_{k=0}^{l} \hat{g}_{l-k,+}(n) \lambda^{k}, \\
& \widehat{G}_{0,-}=-\frac{1}{2}, \quad \widehat{G}_{l,-}(\lambda)=\sum_{k=0}^{l} \hat{g}_{l-k,-}(n) \lambda^{-k} . \\
& \widehat{H}_{0, \pm}=0, \quad \widehat{H}_{l,+}(\lambda)=\sum_{k=0}^{l-1} \hat{h}_{l-k,+}(n) \lambda^{k}, \quad \widehat{H}_{l,-}(\lambda)=\sum_{k=1}^{l} \hat{h}_{l-k,-}(n) \lambda^{-k} .
\end{aligned}
$$

Then one immediately finds that

$$
\begin{aligned}
& \widetilde{F}_{\underline{m}}(n)=\sum_{k=0}^{m} \tilde{\alpha}_{m-k,+} \widehat{F}_{k,+}+\sum_{k=0}^{m-1} \tilde{\alpha}_{m-k,+} \widehat{F}_{k,-}, \\
& \widetilde{G}_{\underline{m}}(n)=\sum_{k=0}^{m} \tilde{\alpha}_{m-k,+} \widehat{G}_{k,+}+\sum_{k=0}^{m} \tilde{\alpha}_{m-k,+} \widehat{G}_{k,-}, \\
& \widetilde{H}_{\underline{m}}(n)=\sum_{k=0}^{m} \tilde{\alpha}_{m-k,+} \widehat{H}_{k,+}+\sum_{k=0}^{m-1} \tilde{\alpha}_{m-k,+} \widehat{H}_{k,-} .
\end{aligned}
$$


Noticing the first expression of (3.3), (3.5) and (3.6), we can write $F(n)$ and $H(n)$ as finite products which take the form:

$$
\begin{aligned}
& F(n)=\alpha_{0,+} \varepsilon u \lambda^{-N_{2}} \prod_{j=1}^{N}\left(\lambda-\mu_{j}(n)\right), \\
& H(n)=\alpha_{0,+} \varepsilon v^{-} \lambda^{-N_{2}} \prod_{j=1}^{N}\left(\lambda-v_{j}(n)\right), \quad N=N_{1}+N_{2}-1,
\end{aligned}
$$

where the roots $\left\{\mu_{j}(n)\right\}_{j=1, \ldots, N},\left\{v_{j}(n)\right\}_{j=1, \ldots, N}$ are called elliptic variables. Comparing the coefficients of $\lambda^{N_{1}-1}, \lambda^{-N_{2}}$ in the expressions of $F(n)$ in (3.6) and (3.9), respectively, we arrive at

$$
\begin{aligned}
& \sum_{j=1}^{N} \mu_{j}(n)=\frac{\varepsilon u\left(u v+u v^{-}\right)-v}{\varepsilon u}-\frac{\alpha_{1,+}}{\alpha_{0,+}} \\
& \sum_{j=1}^{N} v_{j}(n)=\frac{\varepsilon v^{-}\left(u v^{-}+u^{-} v^{-}\right)-u^{-}}{\varepsilon v^{-}}-\frac{\alpha_{1,+}}{\alpha_{0,+}}, \\
& \frac{\varepsilon v^{-}}{u}=(-1)^{N} \frac{\alpha_{0,+}}{\alpha_{0,-}} \prod_{j=1}^{N} \mu_{j}(n), \\
& \frac{\varepsilon u}{v^{-}}=(-1)^{N} \frac{\alpha_{0,+}}{\alpha_{0,-}} \prod_{j=1}^{N} v_{j}(n) .
\end{aligned}
$$

Since $\operatorname{det} W_{n}$ only depends on $\lambda$, whose coefficients are constants of the $n$-flow and $t_{\underline{m}}$-flow, we have

$$
-\operatorname{det} W_{n}=G^{2}(n)+\lambda F(n) H(n)=\frac{1}{4} \alpha_{0,+}^{2} \lambda^{-2 N_{2}} \prod_{j=1}^{2 N+2}\left(\lambda-\lambda_{j}\right)=R(\lambda),
$$

from which we obtain

$$
\left.G(n)\right|_{\lambda=\mu_{k}(n)}=\sqrt{R\left(\mu_{k}(n)\right)},\left.\quad G(n)\right|_{\lambda=v_{k}(n)}=\sqrt{R\left(v_{k}(n)\right)} .
$$

Noticing (3.4) and (3.9), we get

$$
\begin{aligned}
& \left.F(n)_{t_{\underline{m}}}\right|_{\lambda=\mu_{k}(n)}=-\alpha_{0,+} \varepsilon u \mu_{k, t_{\underline{m}}}(n) \mu_{k}(n)^{-N_{2}} \prod_{\substack{j=1 \\
j \neq k}}^{N}\left(\mu_{k}(n)-\mu_{j}(n)\right)=-\left.2 \sqrt{R\left(\mu_{k}(n)\right)} \widetilde{F}_{\underline{m}}(n)\right|_{\lambda=\mu_{k}(n),}, \\
& \left.H(n)_{t_{\underline{m}}}\right|_{\lambda=v_{k}(n)}=-\alpha_{0,+} \varepsilon v^{-} v_{k, t_{\underline{m}}}(n) v_{k}(n)^{-N_{2}} \prod_{\substack{j=1 \\
j \neq k}}^{N}\left(v_{k}(n)-v_{j}(n)\right)=\left.2 \sqrt{R\left(v_{k}(n)\right)} \widetilde{H}_{\underline{m}}(n)\right|_{\lambda=v_{k}(n),},
\end{aligned}
$$

which means

$$
\begin{aligned}
& \mu_{k, \underline{t_{\underline{m}}}}(n)=\frac{\left.2 \sqrt{R\left(\mu_{k}(n)\right)} \mu_{k}^{N_{2}}(n) \widetilde{F}_{\underline{m}}(n)\right|_{\lambda=\mu_{k}(n)}}{\alpha_{0,+} \varepsilon u \prod_{\substack{j=1 \\
j \neq k}}^{N}\left(\mu_{k}(n)-\mu_{j}(n)\right)} \\
& v_{k, t_{\underline{m}}}(n)=-\frac{\left.2 \sqrt{R\left(v_{k}(n)\right)} v_{k}^{N_{2}}(n) \widetilde{H}_{\underline{m}}(n)\right|_{\lambda=v_{k}(n)}}{\alpha_{0,+} \varepsilon v^{-} \prod_{\substack{j=1 \\
j \neq k}}^{N}\left(v_{k}(n)-v_{j}(n)\right)}
\end{aligned}
$$


with $1 \leq k \leq N$ and

$$
\begin{aligned}
& \left.\widetilde{F}_{\underline{m}}(n)\right|_{\lambda=\mu_{k}}(n)=\sum_{j=0}^{m} \tilde{f}_{j,+}(n) \mu_{k}^{m-j}+\sum_{j=0}^{m-1} \tilde{f}_{j,-}(n) \mu_{k}^{-m+j}, \\
& \left.\widetilde{H}_{\underline{m}}(n)\right|_{\lambda=v_{k}}(n)=\sum_{j=0}^{m} \tilde{h}_{j,+}(n) v_{k}^{m-j}+\sum_{j=0}^{m-1} \tilde{h}_{j,-}(n) v_{k}^{-m+j} .
\end{aligned}
$$

\section{Straightening out of various flows}

In the section, we shall discuss straightening out of the corresponding continuous flows. Noticing (3.11), one is naturally led to introduce the hyperelliptic curve $\mathscr{K}_{N}$ of arithmetic genus $N$ defined by

$$
\mathscr{K}_{N}: \quad \mathscr{F}_{N}(\lambda, y)=y^{2}-4 \alpha_{0,+}^{-2} \lambda^{2 N_{2}} R(\lambda)=0,
$$

where $\left\{\lambda_{j}\right\}_{j=1, \cdots, 2 N+2} \subset \mathbb{C}$ and

$$
R(\lambda)=\frac{1}{4} \alpha_{0,+}^{2} \lambda^{-2 N_{2}} \prod_{j=1}^{2 N+2}\left(\lambda-\lambda_{j}\right)
$$

The curve $\mathscr{K}_{N}$ can be compactified by joining two points at infinity, $P_{\infty \pm}$, where $P_{\infty+} \neq P_{\infty-}$. For notational simplicity the compactification is also denoted by $\mathscr{K}_{N}$. Points $P$ on $\mathscr{K}_{N} \backslash\left\{P_{\infty+}, P_{\infty-}\right\}$ are represented as pairs $P=(\lambda, y)$, where $y(\cdot)$ is the meromorphic function on $\mathscr{K}_{N}$ satisfying $\mathscr{F}_{N}(\lambda, y)=0$. Here we assume that the zeros $\lambda_{j}$ of $R(\lambda)$ in (3.11) are mutually distinct, which means $\lambda_{j} \neq \lambda_{k}$, for $j \neq k, 1 \leq j, k \leq 2 N+2$, then the hyperelliptic curve $\mathscr{K}_{N}$ becomes nonsingular. According to the definition of $\mathscr{K}_{N}$, we can lift the roots $\left\{\mu_{j}(n)\right\}$ of $F(n)$ and $\left\{v_{j}(n)\right\}$ of $H(n)$ to $\mathscr{K}_{N}$ by introducing

$$
\begin{aligned}
& \hat{\mu}_{j}\left(n, t_{\underline{m}}\right)=\left(\mu_{j}\left(n, t_{\underline{m}}\right),-2 \alpha_{0,+}^{-1} \mu_{j}\left(n, t_{\underline{m}}\right)^{N_{2}} G\left(\mu_{j}\left(n, t_{\underline{m}}\right), n, t_{\underline{m}}\right)\right), \\
& \hat{v}_{j}\left(n, t_{\underline{m}}\right)=\left(v_{j}\left(n, t_{\underline{m}}\right), 2 \alpha_{0,+}^{-1} v_{j}\left(n, t_{\underline{m}}\right)^{N_{2}} G\left(v_{j}\left(n, t_{\underline{m}}\right), n, t_{\underline{m}}\right)\right),
\end{aligned}
$$

where $j=1, \ldots, N,\left(n, t_{\underline{m}}\right) \in \mathbb{Z} \times \mathbb{R}$. We also introduce the points $P_{0, \pm}$ by

$$
P_{0, \pm}=(0, y(\lambda=0))=\left(0, \pm \frac{\alpha_{0,-}}{\alpha_{0,+}}\right) \in \mathscr{K}_{N}, \quad \frac{\alpha_{0,-}^{2}}{\alpha_{0,+}^{2}}=\prod_{j=1}^{2 N+2} \lambda_{j} .
$$

We emphasize that $P_{0, \pm}$ and $P_{\infty \pm}$ are not necessarily on the same sheet of $\mathscr{K}_{N}$.

Next, we briefly recall our conventions used in connection with divisors on $\mathscr{K}_{N}$. A map, $D$ : $\mathscr{K}_{N} \rightarrow \mathbb{Z}$, is called a divisor on $\mathscr{K}_{N}$ if $D(P) \neq 0$ for only finitely many $P \in \mathscr{K}_{N}$. The set of divisors on $\mathscr{K}_{N}$ is denote by $\operatorname{Div}\left(\mathscr{K}_{N}\right)$. We shall employ the following notation for divisors,

$$
\begin{gathered}
D_{Q_{0} \underline{Q}}=D_{\underline{Q}}+D_{Q_{0}}, \quad D_{\underline{Q}}=D_{Q_{1}}+\cdots+D_{Q_{N}}, \\
\underline{Q}=\left\{Q_{1}, \ldots, Q_{N}\right\} \in \operatorname{Sym}^{N}\left(\mathscr{K}_{N}\right), \quad Q_{0} \in \mathscr{K}_{N}, \quad N \in \mathbb{N},
\end{gathered}
$$

where for any $Q \in \mathscr{K}_{N}$,

$$
D: \mathscr{K}_{N} \rightarrow \mathbb{N}_{0}, \quad P \mapsto D_{Q}(P)= \begin{cases}1, & \text { for } P=Q, \\ 0, & P \in \mathscr{K}_{N} \backslash\{Q\},\end{cases}
$$


and $\operatorname{Sym}^{N}\left(\mathscr{K}_{N}\right)$ denotes the $N$ th symmetric product of $\mathscr{K}_{N}$.

From (4.1) we know that

$$
\frac{1}{4} \alpha_{0,+}^{2} \lambda^{-2 N_{2}} y^{2}=G^{2}+\lambda F H
$$

that is

$$
\left(\frac{1}{2} \alpha_{0,+} \lambda^{-N_{2}} y+G\right)\left(\frac{1}{2} \alpha_{0,+} \lambda^{-N_{2}} y-G\right)=\lambda F H,
$$

then we can define the fundamental meromorphic function $\phi\left(\cdot, n, t_{\underline{m}}\right)$ on $\mathscr{K}_{N}$ by

$$
\begin{gathered}
\phi\left(P, n, t_{\underline{m}}\right)=\frac{\frac{1}{2} \alpha_{0,+} \lambda^{-N_{2}} y-G\left(n, t_{\underline{m}}\right)}{F\left(n, t_{\underline{m}}\right)}=\frac{\lambda H\left(n, t_{\underline{m}}\right)}{\frac{1}{2} \alpha_{0,+} \lambda^{-N_{2}} y+G\left(n, t_{\underline{m}}\right)}, \\
P=(\lambda, y) \in \mathscr{K}_{N}, \quad n \in \mathbb{N},
\end{gathered}
$$

with divisor $\left(\phi\left(\cdot, n, t_{\underline{m}}\right)\right)$ of $\phi\left(\cdot, n, t_{\underline{m}}\right)$ given by

$$
\left(\phi\left(\cdot, n, t_{\underline{m}}\right)\right)=D_{P_{0,-\underline{\hat{v}}}\left(n, t_{\underline{\underline{m}}}\right)}-D_{P_{\infty} \underline{\hat{\mu}}\left(n, t_{\underline{\underline{m}}}\right)}
$$

using (3.9). Here we abbreviated

$$
\underline{\hat{\mu}}\left(n, t_{\underline{m}}\right)=\left\{\hat{\mu}_{1}\left(n, t_{\underline{m}}\right), \ldots, \hat{\mu}_{N}\left(n, t_{\underline{m}}\right)\right\}, \quad \underline{\hat{v}}\left(n, t_{\underline{m}}\right)=\left\{\hat{v}_{1}\left(n, t_{\underline{m}}\right), \ldots, \hat{v}_{N}\left(n, t_{\underline{m}}\right)\right\} .
$$

In order to straighten out the corresponding flows, we consider the Riemann surface $\mathscr{K}_{N}$ and equip $\mathscr{K}_{N}$ with canonical basis cycles: $a_{1}, \cdots, a_{N} ; b_{1}, \cdots, b_{N}$, which are independent and have intersection numbers as follows

$$
a_{i} \circ a_{j}=0, \quad b_{i} \circ b_{j}=0, \quad a_{i} \circ b_{j}=\delta_{i j} .
$$

For the present, we will choose our basis as the following set

$$
\tilde{\omega}_{l}=\frac{\lambda^{l-1} d \lambda}{\sqrt{4 \alpha_{0,+}^{-2} \lambda^{2 N_{2}} R(\lambda)}}, \quad 1 \leq l \leq N
$$

which are $N$ linearly independent homomorphic differentials on $\mathscr{K}_{N}$. By using the cycles $a_{j}$ and $b_{j}$, the period matrices $A$ and $B$ can be constructed from

$$
A_{i j}=\int_{a_{j}} \tilde{\omega}_{i}, \quad B_{i j}=\int_{b_{j}} \tilde{\omega}_{i}
$$

It is possible to show that matrices $A$ and $B$ are invertible $[17,19]$. Now we define the matrices $C$ and $\tau$ by $C=A^{-1}, \tau=A^{-1} B$. The matrix $\tau$ can be shown to be symmetric $\left(\tau_{i j}=\tau_{j i}\right)$ and it has 
positive definite imaginary part $(\operatorname{Im} \tau>0)$. If we normalize $\tilde{\omega}_{l}$ into the new basis $\omega_{j}$

$$
\omega_{j}=\sum_{l=1}^{N} C_{j l} \tilde{\omega}_{l}, \quad 1 \leq j \leq N,
$$

then we have

$$
\int_{a_{i}} \omega_{j}=\sum_{l=1}^{N} C_{j l} \int_{a_{i}} \tilde{\omega}_{l}=\delta_{j i}, \quad \int_{b_{i}} \omega_{j}=\tau_{j i}
$$

Let $\mathscr{T}$ be the lattice generated by $2 N$ vectors $\delta_{j}, \tau_{j}$, where $\delta_{j}=(\underbrace{0, \ldots, 0}_{j-1}, 1, \underbrace{0, \ldots, 0}_{N-j})$ and $\tau_{j}=$ $\tau \delta_{j}$. The complex torus $\mathscr{J}=\mathbb{C}^{N} / \mathscr{T}$ is called Jacobian variety of $\mathscr{K}_{N}$. Now we introduce the Abel $\operatorname{map} \mathscr{A}(P): \operatorname{Div}\left(\mathscr{K}_{N}\right) \rightarrow \mathscr{J}$

$$
\mathscr{A}(P)=\int_{P_{0}}^{P} \underline{\omega}, \quad \mathscr{A}\left(\sum n_{k} P_{k}\right)=\sum n_{k} \mathscr{A}\left(P_{k}\right),
$$

where $P, P_{k} \in \mathscr{K}_{N}, \underline{\omega}=\left(\omega_{1}, \ldots, \omega_{N}\right)$. The Riemann theta function is defined as [17, 19]

$$
\theta(P, D)=\theta(\underline{\Lambda}-\mathscr{A}(P)+\mathscr{A}(D))
$$

with $P \in \mathscr{K}_{N}, D \in \operatorname{Div}\left(\mathscr{K}_{N}\right)$, and $\underline{\Lambda}=\left(\Lambda_{1}, \ldots, \Lambda_{N}\right)$ is defined by

$$
\Lambda_{j}=\frac{1}{2}\left(1+\tau_{j j}\right)-\sum_{\substack{i=1 \\ i \neq j}}^{N} \int_{a_{i}} \omega_{i} \int_{P_{0}}^{P} \omega_{j}, \quad j=1, \cdots, N .
$$

Define the Abel-Jacobi coordinates

$$
\begin{aligned}
& \underline{\rho}^{(1)}\left(n, t_{\underline{m})}=\mathscr{A}\left(\sum_{k=1}^{N} P\left(\mu_{k}(n)\right)\right)=\sum_{k=1}^{N} \mathscr{A}\left(P\left(\mu_{k}(n)\right)\right)=\sum_{k=1}^{N} \int_{P_{0}}^{P\left(\mu_{k}(n)\right)} \underline{\omega},\right. \\
& \underline{\rho}^{(2)}\left(n, t_{\underline{m})}=\mathscr{A}\left(\sum_{k=1}^{N} P\left(v_{k}(n)\right)\right)=\sum_{k=1}^{N} \mathscr{A}\left(P\left(v_{k}(n)\right)\right)=\sum_{k=1}^{N} \int_{P_{0}}^{P\left(v_{k}(n)\right)} \underline{\omega},\right.
\end{aligned}
$$

where

$P\left(\mu_{k}(n)\right)=\left(\mu_{k}(n), \sqrt{4 \alpha_{0,+}^{-2} \mu_{k}(n)^{2 N_{2}} R\left(\mu_{k}(n)\right)}\right), P\left(v_{k}(n)\right)=\left(v_{k}(n), \sqrt{4 \alpha_{0,+}^{-2} v_{k}(n)^{2 N_{2}} R\left(v_{k}(n)\right)}\right)$, and $P_{0}$ is chosen as a base point on $\mathscr{K}_{N}$. The components of the Abel-Jacobi coordinates in (4.11) read

$$
\begin{aligned}
& \rho_{j}^{(1)}\left(n, t_{\underline{m}}\right)=\sum_{k=1}^{N} \int_{P_{0}}^{\hat{\mu}_{k}\left(n, t_{\underline{m}}\right)} \omega_{j}=\sum_{k=1}^{N} \sum_{l=1}^{N} C_{j l} \int_{\lambda\left(P_{0}\right)}^{\mu_{k}} \frac{\lambda^{l-1} d \lambda}{\sqrt{4 \alpha_{0,+}^{-2} \lambda^{2 N_{2}} R(\lambda)}}, \\
& \rho_{j}^{(2)}\left(n, t_{\underline{m}}\right)=\sum_{k=1}^{N} \int_{P_{0}}^{\hat{v}_{k}\left(n, t_{\underline{m}}\right)} \omega_{j}=\sum_{k=1}^{N} \sum_{l=1}^{N} C_{j l} \int_{\lambda\left(P_{0}\right)}^{v_{k}} \frac{\lambda^{l-1} d \lambda}{\sqrt{4 \alpha_{0,+}^{-2} \lambda^{2 N_{2} R(\lambda)}}},
\end{aligned}
$$

where $1 \leq j \leq N$. Without loss of generality, we choose the branch point $P_{0}=\left(\lambda_{j_{0}}, 0\right), j_{0} \in$ $\{1, \cdots, 2 N+2\}$, as a convenient base point on $\mathscr{K}_{N}$, and $\lambda\left(P_{0}\right)$ is its local coordinate. Then according 
to (4.9) and the definition of Riemann theta function in (4.10), we have

$$
\begin{aligned}
& \theta\left(P, D_{\hat{\underline{\mu}}\left(n, t_{\underline{m}}\right)}\right)=\theta\left(\underline{\Lambda}-\mathscr{A}(P)+\underline{\rho}^{(1)}\left(n, t_{\underline{m}}\right)\right), \\
& \theta\left(P, D_{\underline{\hat{\hat{v}}}\left(n, t_{\underline{m}}\right)}\right)=\theta\left(\underline{\Lambda}-\mathscr{A}(P)+\underline{\rho}^{(2)}\left(n, \underline{t}_{\underline{m}}\right)\right) .
\end{aligned}
$$

From (3.14), we obtain

$$
\begin{aligned}
& \partial_{\underline{t_{\underline{m}}}} \rho_{j}^{(1)}\left(n, t_{\underline{m}}\right)=\sum_{l=1}^{N} \sum_{k=1}^{N} C_{j l} \frac{\mu_{k}^{l-1} \mu_{k, t_{\underline{m}}}}{\sqrt{4 \alpha_{0,+}^{-2} \mu_{k}(n)^{2 N_{2}} R\left(\mu_{k}\right)}}=\sum_{l=1}^{N} \sum_{k=1}^{N} \frac{\left.C_{j l} \mu_{k}^{l-1} \widetilde{F}_{\underline{m}}(n)\right|_{\lambda=\mu_{k}}}{\varepsilon u \prod_{\substack{i=1 \\
i \neq k}}^{N}\left(\mu_{k}-\mu_{i}\right)} \\
& \partial_{t_{\underline{m}}} \rho_{j}^{(2)}\left(n, t_{\underline{m}}\right)=\sum_{l=1}^{N} \sum_{k=1}^{N} C_{j l} \frac{v_{k}^{l-1} v_{k, t_{\underline{m}}}}{\sqrt{4 \alpha_{0,+}^{-2} v_{k}(n)^{2 N_{2}} R\left(v_{k}\right)}}=\sum_{l=1}^{N} \sum_{k=1}^{N} \frac{\left.\left(-C_{j l}\right) v_{k}^{l-1} \widetilde{H}_{\underline{m}}(n)\right|_{\lambda=v_{k}}}{\varepsilon v^{-} \prod_{\substack{i=1 \\
i \neq k \\
i \neq k}}^{N}\left(v_{k}-v_{i}\right)} .
\end{aligned}
$$

Before discussing the following theorem, we begin with some elementary results. Let

$$
\left\{\lambda_{j}\right\}_{j=1, \ldots, 2 N+2} \subset \mathbb{C}
$$

for some $N \in \mathbb{N}_{0}$ and $\xi \subset \mathbb{C}$, such that $|\xi|<\min \left\{\left|\lambda_{1}\right|^{-1}, \ldots,\left|\lambda_{2 N+2}\right|^{-1}\right\}$, and abbreviate

$$
\underline{\lambda}=\left(\lambda_{1}, \ldots, \lambda_{2 N+2}\right), \quad \underline{\lambda}^{-1}=\left(\lambda_{1}^{-1}, \ldots, \lambda_{2 N+2}^{-1}\right) .
$$

Then

$$
\left(\prod_{j=1}^{2 N+2}\left(1-\lambda_{j} \xi\right)\right)^{-\frac{1}{2}}=\sum_{k=0}^{\infty} \hat{\alpha}_{k}(\underline{\lambda}) \xi^{k}
$$

where

$$
\begin{aligned}
& \hat{\alpha}_{0}(\underline{\lambda})=1, \\
& \hat{\alpha}_{k}(\underline{\lambda})=\sum_{\substack{j_{1}, \ldots, j_{2 N+2}=0 \\
j_{1}+\ldots+j_{2 N+2}=k}}^{k} \frac{\left(2 j_{1}\right) ! \ldots\left(2 j_{2 N+2}\right) !}{2^{2 k}\left(j_{1} !\right)^{2} \ldots\left(j_{2 N+2} !\right)^{2}} \lambda_{1}^{j_{1}} \ldots \lambda_{2 N+2}^{j_{2 N+2}}, \quad k \in \mathbb{N} .
\end{aligned}
$$

The first three coefficients are given explicitly by

$$
\begin{aligned}
& \hat{\alpha}_{0}(\underline{\lambda})=1, \quad \hat{\alpha}_{1}(\underline{\lambda})=\frac{1}{2} \sum_{j=1}^{2 N+2} \lambda_{j}, \\
& \hat{\alpha}_{2}(\underline{\lambda})=\frac{1}{4} \sum_{\substack{j, k=1 \\
j<k}}^{2 N+2} \lambda_{j} \lambda_{k}+\frac{3}{8} \sum_{j=1}^{2 N+2} \lambda_{j}^{2} .
\end{aligned}
$$

In a similar way, we have

$$
\left(\prod_{j=1}^{2 N+2}\left(1-\lambda_{j} \xi\right)\right)^{\frac{1}{2}}=\sum_{k=0}^{\infty} \alpha_{k}(\underline{\lambda}) \xi^{k}
$$

where

$$
\begin{aligned}
& \alpha_{0}(\underline{\lambda})=1, \\
& \alpha_{k}(\underline{\lambda})=\sum_{\substack{j_{1}, \ldots, j_{2 N+2}=0 \\
j_{1}+\ldots+j_{2 N+2}=k}}^{k} \frac{\left(2 j_{1}\right) ! \ldots\left(2 j_{2 N+2}\right) !}{2^{2 k}\left(j_{1} !\right)^{2} \ldots\left(j_{2 N+2} !\right)^{2}\left(2 j_{1}-1\right) \ldots\left(2 j_{2 N+2}-1\right)} \lambda_{1}^{j_{1}} \ldots \lambda_{2 N+2}^{j_{2 N+2}}, \quad k \in \mathbb{N} .
\end{aligned}
$$


The first few coefficients explicitly read

$$
\begin{aligned}
& \alpha_{0}(\underline{\lambda})=1, \quad \alpha_{1}(\underline{\lambda})=-\frac{1}{2} \sum_{j=1}^{2 N+2} \lambda_{j}, \\
& \alpha_{2}(\underline{\lambda})=\frac{1}{4} \sum_{\substack{j, k=1 \\
j<k}}^{2 N+2} \lambda_{j} \lambda_{k}-\frac{1}{8} \sum_{j=1}^{2 N+2} \lambda_{j}^{2} .
\end{aligned}
$$

Next, we turn to asymptotic expansions of various quantities in connection with the selfdual network hierarchy. we begin with some general results associated with the self-dual network hierarchy. Considering a fundamental system of solutions $\Psi_{ \pm}(\lambda, \cdot)=\left(\psi_{1, \pm}(\lambda, \cdot), \psi_{2, \pm}(\lambda, \cdot)\right)^{T}$ of $U(\lambda) \Psi_{ \pm}(\lambda)=\Psi_{ \pm}^{+}(\lambda)$ for $\lambda \in \mathbb{C}$, with $U$ given by (2.1) such that

$$
\operatorname{det}\left(\Psi_{-}(\lambda), \Psi_{+}(\lambda)\right) \neq 0 .
$$

Introducing

$$
\phi_{ \pm}=\frac{\psi_{2, \pm}}{\psi_{1, \pm}}
$$

then $\phi_{ \pm}$satisfy the Riccati-type equation

$$
(\lambda+u v) \phi_{ \pm}^{+}+\left(\lambda^{-1} v+\varepsilon u\right) \phi_{ \pm}^{+} \phi_{ \pm}=u+\lambda \varepsilon v+\left(\lambda^{-1}+u v\right) \phi_{ \pm},
$$

and one introduces in addition

$$
\begin{aligned}
\check{F} & =\frac{2}{\phi_{+}-\phi_{-}}, \\
\check{G} & =-\frac{\phi_{+}+\phi_{-}}{\phi_{+}-\phi_{-}}, \\
\check{H} & =\frac{-2 \lambda^{-1} \phi_{+} \phi_{-}}{\phi_{+}-\phi_{-}} .
\end{aligned}
$$

Using the Riccati-type equation (4.20), one derives the identities

$$
\begin{aligned}
& (\lambda+u v)\left(\check{G}^{+}-\check{G}\right)+(u+\lambda \varepsilon v) \check{F}^{+}-(\lambda \varepsilon u+v) \check{H}=0, \\
& (\varepsilon u+\lambda-1 v)\left(\check{G}^{+}+\check{G}\right)+(\lambda-1+u v) \check{F}^{+}-(\lambda+u v) \check{F}=0, \\
& (u+\lambda \varepsilon v)\left(\check{G}^{+}+\breve{G}\right)-\lambda(\lambda+u v) \check{H}^{+}+(1+\lambda u v) \check{H}=0, \\
& (\lambda-1+u v)\left(\check{G}^{+}-\breve{G}\right)-(\lambda \varepsilon u+v) \check{H}^{+}+(u+\lambda \varepsilon v) \check{F}=0, \\
& \check{G}^{2}+\lambda \check{F} \breve{H}=1 .
\end{aligned}
$$

Moreover, (4.24) also permit one to derive nonlinear difference equations for $\check{G}, \check{F}, \check{H}$, separately,

$$
\begin{aligned}
& \left\{\left(\gamma^{-} \pi^{-}\right)^{2}(v+\lambda \varepsilon u)\left(\lambda^{-1} v+\varepsilon u\right) \check{F}^{-}+\left[(\lambda+u v)^{2}\left(v^{-}+\lambda \varepsilon u^{-}\right)\left(\lambda^{-1} v^{-}+\varepsilon u^{-}\right)\right.\right. \\
& \left.\left.-\left(\lambda^{-1}+u^{-} v^{-}\right)^{2}(v+\lambda \varepsilon u)\left(\lambda^{-1} v+\varepsilon u\right)\right] F-\gamma^{2} \pi^{2}\left(v^{-}+\lambda \varepsilon u^{-}\right)\left(\lambda^{-1} v^{-}+\varepsilon u^{-}\right) \check{F}^{+}\right\}^{2} \\
& +4 \lambda\left(\lambda^{-1} v+\varepsilon u\right)\left(\lambda^{-1} v^{-}+\varepsilon u^{-}\right)\left[\left(\lambda^{-1}+u^{-} v^{-}\right)(v+\lambda \varepsilon u)+(\lambda+u v)\left(v^{-}+\lambda \varepsilon u^{-}\right)\right] \breve{F} \\
& \times\left\{(\lambda+u v)\left(\lambda^{-1}+u^{-} v^{-}\right)\left[(\lambda+u v)\left(\lambda^{-1} v^{-}+\varepsilon u^{-}\right)+\left(\lambda^{-1} v+\varepsilon u\right)\left(\lambda{ }^{-1}+u^{-} v^{-}\right)\right] \breve{F}\right. \\
& \left.-\gamma^{2} \pi^{2}\left(\lambda^{-1}+u^{-} v^{-}\right)\left(\lambda^{-1} v^{-}+\varepsilon u^{-}\right) \check{F}^{+}-\left(\gamma^{-} \pi^{-}\right)^{2}(\lambda+u v)\left(\lambda^{-1} v+\varepsilon u\right) \check{F}^{-}\right\} \\
& =4\left(\lambda^{-1} v^{-}+\varepsilon u^{-}\right)^{2}\left(\lambda^{-1} v+\varepsilon u\right)^{2}\left[(v+\lambda \varepsilon u)\left(\lambda^{-1}+u^{-} v^{-}\right)+(\lambda+u v)\left(v^{-}+\lambda \varepsilon u^{-}\right)\right]^{2},
\end{aligned}
$$




$$
\begin{aligned}
& {\left[(\lambda+u v)\left(\lambda+u^{-} v^{-}\right)(u+\lambda \varepsilon v)\left(v^{-}+\lambda \varepsilon u^{-}\right)-(v+\lambda \varepsilon u)\left(\lambda^{-1}+u^{-} v^{-}\right)\left(\lambda^{-1}+u v\right)\left(u^{-}+\lambda \varepsilon v^{-}\right)\right]^{2} G^{2}} \\
& +\left\{(u+\lambda \varepsilon v)\left(v^{-}+\lambda \varepsilon u^{-}\right)\left(\lambda+u^{-} v^{-}\right)\left(\lambda^{-1} v+\varepsilon u\right)\left(G^{+}+G\right)\right. \\
& +\left(u^{-}+\lambda \varepsilon v^{-}\right)(v+\lambda \varepsilon u)\left(\lambda^{-1} v^{-}+\varepsilon u^{-}\right)\left(\lambda^{-1}+u v\right)\left(G+G^{-}\right) \\
& +\left(\lambda^{-1}+u^{-} v^{-}\right)(v+\lambda \varepsilon u)\left(\lambda^{-1}+u v\right)\left(\lambda+u^{-} v^{-}\right)\left(G-G^{-}\right) \\
& \left.-(\lambda+u v)\left(\lambda+u^{-} v^{-}\right)\left(\lambda^{-1}+u v\right)\left(v^{-}+\lambda \varepsilon u^{-}\right)\left(G^{+}-G\right)\right\} \\
& \times\left\{(v+\lambda \varepsilon u)\left(\lambda^{-1}+u^{-} v^{-}\right)(u+\lambda \varepsilon v)\left(u^{-}+\lambda \varepsilon v^{-}\right)\left(G^{+}+G\right)\right. \\
& +\left(v^{-}+\lambda \varepsilon u^{-}\right)(u+\lambda \varepsilon v)(\lambda+u v)\left(u^{-}+\lambda \varepsilon v^{-}\right)\left(G+G^{-}\right) \\
& +\left(\lambda+u^{-} v^{-}\right)(\lambda+u v)(u+\lambda \varepsilon v)\left(1+\lambda u^{-} v^{-}\right)\left(G-G^{-}\right) \\
& \left.-\left(\lambda^{-1}+u v\right)\left(u^{-}+\lambda \varepsilon v^{-}\right)(\lambda+u v)\left(1+\lambda u^{-} v^{-}\right)\left(G^{+}-G\right)\right\} \\
& =\left[(\lambda+u v)\left(\lambda+u^{-} v^{-}\right)(u+\lambda \varepsilon v)\left(v^{-}+\lambda \varepsilon u^{-}\right)-(v+\lambda \varepsilon u)\left(\lambda^{-1}+u^{-} v^{-}\right)\left(\lambda^{-1}+u v\right)\left(u^{-}+\lambda \varepsilon v^{-}\right)\right]^{2} \text {, } \\
& \left\{\left[\lambda\left(\lambda+u^{-} v^{-}\right)^{2}(u+\lambda \varepsilon v)^{2}-(1+\lambda u v)\left(\lambda^{-1}+u v\right)\left(u^{-}+\lambda \varepsilon v^{-}\right)^{2}\right] \check{H}\right. \\
& \left.+\lambda(\gamma \pi)^{2}\left(u^{-}+\lambda \varepsilon v^{-}\right)^{2} \check{H}^{+}-\lambda\left(\gamma^{-} \pi^{-}\right)^{2}(u+\lambda \varepsilon v)^{2} \check{H}^{-}\right\}^{2}+4 \lambda\left[(u+\lambda \varepsilon v)^{2}\left(\lambda+u^{-} v^{-}\right)\left(u^{-}+\lambda \varepsilon v^{-}\right)\right. \\
& \left.+\left(u^{-}+\lambda \varepsilon v^{-}\right)^{2}(u+\lambda \varepsilon v)\left(\lambda^{-1}+u v\right)\right] \check{H}\left\{\left[(1+\lambda u v)\left(\lambda^{-1}+u v\right)\left(\lambda+u^{-} v^{-}\right)\left(u^{-}+\lambda \varepsilon v^{-}\right)\right.\right. \\
& \left.+\lambda\left(\lambda+u^{-} v^{-}\right)^{2}\left(\lambda^{-1}+u v\right)(u+\lambda \varepsilon v)\right] \check{H}-\lambda(\gamma \pi)^{2}\left(\lambda+u^{-} v^{-}\right)\left(u^{-}+\lambda \varepsilon v^{-}\right) \check{H}^{+} \\
& \left.-\lambda\left(\gamma^{-} \pi^{-}\right)^{2}(u+\lambda \varepsilon v)\left(\lambda^{-1}+u v\right) \check{H}^{-}\right\} \\
& =4\left[(u+\lambda \varepsilon v)^{2}\left(\lambda+u^{-} v^{-}\right)\left(u^{-}+\lambda \varepsilon v^{-}\right)+\left(u^{-}+\lambda \varepsilon v^{-}\right)^{2}(u+\lambda \varepsilon v)\left(\lambda^{-1}+u v\right)\right]^{2} \text {. }
\end{aligned}
$$

Next, we assume the existence of the asymptotic expansions of $\check{F}, \quad \check{G}, \quad \check{H}$ as $P \rightarrow P_{\infty \pm}$ and $P \rightarrow$ $P_{0, \pm}$. More precisely, near $\frac{1}{\lambda}=0$ we assume that

$$
\check{F}(\lambda) \underset{\substack{|\lambda| \rightarrow 0 \\ \lambda \in C_{R}}}{=} \mp \sum_{l=0}^{\infty} \check{f}_{l,+} \lambda^{-l}, \quad \check{G}(\lambda) \underset{\substack{|\lambda| \rightarrow 0 \\ \lambda \in C_{R}}}{=} \mp \sum_{l=0}^{\infty} \check{g}_{l,+} \lambda^{-l}, \quad \check{H}(\lambda) \underset{\substack{|\lambda| \rightarrow 0 \\ \lambda \in C_{R}}}{=} \mp \sum_{l=0}^{\infty} \check{h}_{l,+} \lambda^{-l}, \quad l \in \mathbb{N}_{0},
$$

for $\lambda$ in some cone $C_{R}$ with apex at $\lambda=0$ and some opening angle in $(0,2 \pi]$, exterior to a disk centered at $\lambda=0$ of Sufficiently large radius $R>0$, for some set of coefficients $\breve{f}_{l,+}, \breve{g}_{l,+}, \breve{h}_{l,+}, \quad l \in$ $\mathbb{N}_{0}$, and the sign depends on whether $P \rightarrow P_{\infty+}$ or $P \rightarrow P_{\infty-}$. Similarly, near $\lambda=0$ we assume that

$$
\check{F}(\lambda) \underset{\substack{|\lambda| \rightarrow 0 \\ \lambda \in C_{r}}}{=} \pm \sum_{l=0}^{\infty} \check{f}_{l,-} \lambda^{l}, \quad \check{G}(\lambda) \underset{\substack{|\lambda| \rightarrow 0 \\ \lambda \in C_{r}}}{=} \pm \sum_{l=0}^{\infty} \check{g}_{l,-} \lambda^{l}, \quad \check{H}(\lambda) \underset{\substack{|\lambda| \rightarrow 0 \\ \lambda \in C_{r}}}{=} \pm \sum_{l=0}^{\infty} \check{h}_{l,-} \lambda^{l}, \quad l \in \mathbb{N}_{0},
$$

for $\lambda$ in some cone $C_{r}$ with apex at $\lambda=0$ and some opening angle in $(0,2 \pi]$, interior to a disk centered at $\lambda=0$ of Sufficiently small radius $r>0$, for some set of coefficients $\breve{f}_{l,-}, \check{g}_{l,-}, \breve{h}_{l,-}, \quad l \in$ $\mathbb{N}_{0}$, and the sign depends on whether $P \rightarrow P_{0,+}$ or $P \rightarrow P_{0,-}$. Then we can prove the following result

Theorem 4.1. Suppose $u, v \in \mathbb{C}^{\mathbb{Z}}, u(n) v(n) \neq 0, n \in \mathbb{Z}$, and the existence of the asymptotic expansions (4.28) and (4.29). Then $\check{F}(\lambda), \breve{G}(\lambda), \breve{H}(\lambda)$ have the following asymptotic expansions as $|\lambda| \rightarrow \infty, \lambda \in C_{R}$, respectively, $|\lambda| \rightarrow 0, \lambda \in C_{r}$,

$$
\check{F}(\lambda) \underset{\substack{|\lambda| \rightarrow 0 \\ \lambda \in C_{R}}}{=} \mp \sum_{l=0}^{\infty} \hat{f}_{l,+} \lambda^{-l}, \quad \check{G}(\lambda) \underset{\substack{|\lambda| \rightarrow 0 \\ \lambda \in C_{R}}}{=} \mp \sum_{l=0}^{\infty} \hat{g}_{l,+} \lambda^{-l}, \quad \check{H}(\lambda) \underset{\substack{|\lambda| \rightarrow 0 \\ \lambda \in C_{R}}}{=} \mp \sum_{l=0}^{\infty} \hat{h}_{l,+} \lambda^{-l}, \quad l \in \mathbb{N}_{0},
$$


and

$$
\check{F}(\lambda) \underset{\substack{|\lambda| \rightarrow 0 \\ \lambda \in C_{r}}}{=} \pm \sum_{l=0}^{\infty} \hat{f}_{l,-} \lambda^{l}, \quad \check{G}(\lambda) \underset{\substack{|\lambda| \rightarrow 0 \\ \lambda \in C_{r}}}{=} \pm \sum_{l=0}^{\infty} \hat{g}_{l,-} \lambda^{l}, \quad \check{H}(\lambda) \underset{\substack{\lambda \mid \rightarrow 0 \\ \lambda \in C_{r}}}{=} \pm \sum_{l=0}^{\infty} \hat{h}_{l,-} \lambda^{l}, \quad l \in \mathbb{N}_{0},
$$

where $\hat{f}_{l, \pm}, \quad \hat{g}_{l \pm}, \quad \hat{h}_{l, \pm}$ are the homogeneous versions of the coefficients $f_{l, \pm}, \quad g_{l \pm}, \quad h_{l, \pm}$ defined in (3.11).

Proof. According to (4.28), (4.29), in order to prove (4.30), (4.31), we only need to prove $\breve{f}_{l, \pm}=$ $\hat{f}_{l, \pm}, \breve{g}_{l, \pm}=\hat{g}_{l, \pm}, \breve{h}_{l, \pm}=\hat{h}_{l, \pm}, \quad l \in \mathbb{N}_{0}$. We first consider the expansions (4.30) near $\frac{1}{\lambda}=0$ in detail. Inserting expansions (4.28) for $\check{F}$ into (4.25), expansions (4.28) for $\check{G}$ into (4.26), expansions (4.28) for $\breve{H}$ into (4.27), then yields the nonlinear recursion relations for $\breve{f l}_{l,+}, \breve{g}_{l,+}, \breve{h}_{l,+}$. It's easy to see that there is only pure algebraic computation without inverse operators of difference or differential in the expansions (4.25), (4.26), (4.27), that is, $\breve{f l}_{l,+}, \breve{g}_{l,+}, \breve{h}_{l,+}$ are uniquely determined by the nonlinear recursion relations obtained by comparison with the same coefficients of $\lambda$, respectively. on the other hand, from (2.6)-(2.9), one obtains the same nonlinear relations (4.25), (4.26), (4.27), but with $\breve{F}, \breve{G}, \breve{H}$ replaced by $\widehat{F}, \widehat{G}, \widehat{H}$, respectively. That is, $\breve{f}_{l,+}, \check{g}_{l,+}, \breve{h}_{l,+}$ and $\hat{f}_{l,+}, \hat{g}_{l,+}, \hat{h}_{l,+}$ satisfy the same nonlinear relations, respectively. The signs of $\breve{f}_{1,+}, \breve{g}_{0,+}, \breve{h}_{1,+}$ have been chosen such that the coefficients $\breve{f}_{1,+}=-a^{-}, \quad \check{g}_{0,+}=\frac{1}{2}, \quad \breve{h}_{1,+}=\frac{1}{a} . \breve{f}_{1,+}, \breve{g}_{0,+}, \breve{h}_{1,+}$ are consistent with (3.6) for $\alpha_{0,+}=1$, that is to say, they have the same initial value. Thus, one concludes that

$$
\check{f}_{l,+}=\hat{f}_{l,+}, \quad \check{g}_{l,+}=\hat{g}_{l,+}, \quad \check{h}_{l,+}=\hat{h}_{l,+}, \quad l \in \mathbb{N}_{0} .
$$

In a similar way, we can prove that

$$
\check{f}_{l,-}=\hat{f}_{l,-}, \quad \check{g}_{l,-}=\hat{g}_{l,-}, \quad \check{h}_{l,-}=\hat{h}_{l,-}, l \in \mathbb{N}_{0} .
$$

Given this general result on asymptotic expansions for Laurent polynomial associated with the self-dual network hierarchy, we now specialize to the algebra-geometric case at hand. We recall our convention $y(P)=\mp\left(\zeta^{-N-1}+O\left(\zeta^{-N}\right)\right)$ for $P \rightarrow P_{\infty \pm}\left(\right.$ where $\left.\zeta=\frac{1}{\lambda}\right)$ and $y(P)= \pm \frac{\alpha_{0,-}}{\alpha_{0,+}}+O(\zeta)$ for $P \rightarrow P_{0, \pm}($ where $\zeta=\lambda)$.

Theorem 4.2. Assume $u, v \in \mathbb{C}^{\mathbb{Z}}, u(n) v(n) \neq 0, n \in \mathbb{Z}$, and suppose $P=(\lambda, y) \in$ $\mathscr{K}_{N} \backslash\left\{P_{\infty \pm}, P_{0, \pm}\right\}$. Then $\lambda^{N_{2}} G / y, \quad \lambda^{N_{2}} F / y, \quad \lambda^{N_{2}} H / y$ have the following convergent expansions as $P \rightarrow P_{\infty \pm}$,

$$
\frac{\lambda^{N_{2}}}{\alpha_{0,+}} \frac{G(\lambda)}{y} \underset{\zeta \rightarrow 0}{=} \mp \sum_{l=0}^{\infty} \hat{g}_{l,+} \zeta^{l}, \quad \frac{\lambda^{N_{2}}}{\alpha_{0,+}} \frac{F(\lambda)}{y} \underset{\zeta \rightarrow 0}{=} \mp \sum_{l=0}^{\infty} \hat{f}_{l,+} \zeta^{l}, \quad \frac{\lambda^{N_{2}}}{\alpha_{0},+} \frac{H(\lambda)}{y} \underset{\zeta \rightarrow 0}{=} \mp \sum_{l=0}^{\infty} \hat{h}_{l,+} \zeta^{l},(4
$$

and as $P \rightarrow P_{0, \pm}$,

$$
\frac{\lambda^{N_{2}}}{\alpha_{0,+}} \frac{G(\lambda)}{y} \underset{\zeta \rightarrow 0}{=} \pm \sum_{l=0}^{\infty} \hat{g}_{l,-} \zeta^{l}, \quad \frac{\lambda^{N_{2}}}{\alpha_{0,+}} \frac{F(\lambda)}{y} \underset{\zeta \rightarrow 0}{=} \pm \sum_{l=1}^{\infty} \hat{f}_{l,-} \zeta^{l}, \quad \frac{\lambda^{N_{2}}}{\alpha_{0,+}} \frac{H(\lambda)}{y} \underset{\zeta \rightarrow 0}{=} \pm \sum_{l=1}^{\infty} \hat{h}_{l,-} \zeta^{l} .(4
$$

where $\zeta=\lambda^{-1}($ resp.,$\zeta=\lambda)$ is the local coordinate near $P_{\infty \pm}\left(\operatorname{resp} ., P \rightarrow P_{0, \pm}\right)$, and $\hat{f}_{l, \pm}$, $\hat{g}_{l \pm}, \quad \hat{h}_{l, \pm}$ are the homogeneous versions of the coefficients $f_{l, \pm}, \quad g_{l \pm}, \quad h_{l, \pm}$ as introduced in (3.11). Moreover, one infers for the $\lambda_{j}$-dependent summation constants $\alpha_{l,+}, l=0, \cdots, N_{1}$ and 
$\alpha_{l,-}, l=0, \cdots, N_{2}-1$ in $G(\lambda), F(\lambda), H(\lambda)$ that

$$
\alpha_{k,+}=\alpha_{0,+} \alpha_{k}(\underline{\lambda}), \quad 0 \leq k \leq N_{1} ; \quad \alpha_{k,-}=\alpha_{0,-} \alpha_{k}\left(\underline{\lambda}^{-1}\right), \quad 0 \leq k \leq N_{2}-1
$$

where $\underline{\lambda}=\left(\lambda_{1}, \ldots, \lambda_{2 N+2}\right), \underline{\lambda}^{-1}=\left(\lambda_{1}^{-1}, \ldots, \lambda_{2 N+2}^{-1}\right)$,

$$
\alpha_{0}\left(\underline{\lambda}^{ \pm 1}\right)=1, \quad \alpha_{k}\left(\underline{\lambda}^{ \pm 1}\right)=\sum_{\substack{j_{1}, \ldots, j_{2 N+2}=0 \\ j_{1}+\ldots+j_{2 N+2}=k}}^{k} \frac{\left(2 j_{1}\right) ! \ldots\left(2 j_{2 N+2}\right) ! \lambda_{1}^{ \pm j_{1}} \ldots \lambda_{2 N+2}^{ \pm j_{2 N+2}}}{2^{2 k}\left(j_{1} !\right)^{2} \ldots\left(j_{2 N+2} !\right)^{2}\left(2 j_{1}-1\right) \ldots\left(2 j_{2 N+2}-1\right)}, \quad k \in \mathbb{N} .
$$

In addition, one has the following relations between the homogeneous and nonhomogeneous recursion coefficients:

$$
\begin{aligned}
& f_{l, \pm}=\alpha_{0, \pm} \sum_{k=0}^{l} \alpha_{l-k}\left(\underline{\lambda}^{ \pm 1}\right) \hat{f}_{k, \pm}, \quad l=0, . ., N_{1+\delta_{ \pm}}-\delta_{ \pm}, \\
& g_{l, \pm}=\alpha_{0, \pm} \sum_{k=0}^{l} \alpha_{l-k}\left(\underline{\lambda}^{ \pm 1}\right) \hat{g}_{k, \pm}, \quad l=0, . ., N_{1+\delta_{ \pm}}-\delta_{ \pm} \\
& h_{l, \pm}=\alpha_{0, \pm} \sum_{k=0}^{l} \alpha_{l-k}\left(\underline{\lambda}^{ \pm 1}\right) \hat{h}_{k, \pm}, \quad l=0, . ., N_{1+\delta_{ \pm}}-\delta_{ \pm} \\
& \alpha_{0, \pm} \hat{f}_{l, \pm}=\sum_{k=0}^{l} \hat{\alpha}_{l-k}\left(\underline{\lambda}^{ \pm 1}\right) f_{k, \pm}, \quad l=0, . ., N_{1+\delta_{ \pm}}-\delta_{ \pm} \\
& \alpha_{0, \pm} \hat{g}_{l, \pm}=\sum_{k=0}^{l} \hat{\alpha}_{l-k}\left(\underline{\lambda}^{ \pm 1}\right) g_{k, \pm}, \quad l=0, . ., N_{1+\delta_{ \pm}}-\delta_{ \pm} \\
& \alpha_{0, \pm} \hat{h}_{l, \pm}=\sum_{k=0}^{l} \hat{\alpha}_{l-k}\left(\underline{\lambda}^{ \pm 1}\right) h_{k, \pm}, \quad l=0, . ., N_{1+\delta_{ \pm}}-\delta_{ \pm},
\end{aligned}
$$

where

$$
\hat{\alpha}_{0}\left(\underline{\boldsymbol{\lambda}}^{ \pm 1}\right)=1, \quad \hat{\alpha}_{k}\left(\underline{\boldsymbol{\lambda}}^{ \pm 1}\right)=\sum_{\substack{j_{1}, \ldots, j_{2 N+2}=0 \\ j_{1}+\ldots+j_{2 N+2}=k}}^{k} \frac{\left(2 j_{1}\right) ! \ldots\left(2 j_{2 N+2}\right) ! \lambda_{1}^{ \pm j_{1}} \ldots \lambda_{2 N+2}^{ \pm j_{2 N+2}}}{2^{2 k}\left(j_{1} !\right)^{2} \ldots\left(j_{2 N+2} !\right)^{2}}, \quad k \in \mathbb{N} .
$$

Here we used the convention

$$
\delta_{ \pm}= \begin{cases}0, & +, \\ 1, & -\end{cases}
$$

Proof. Now, we introduce the holomorphic sheet exchange map

$$
*: \mathscr{K}_{N} \rightarrow \mathscr{K}_{N}, \quad P=(\lambda, y) \rightarrow P^{*}=(\lambda,-y), \quad y\left(P^{*}\right)=-y(P), \quad P, P^{*} \in \mathscr{K}_{N} .
$$

Identifying $\phi_{+}(\lambda, \cdot)$ with $\phi(P, \cdot)$ and $\phi_{-}(\lambda, \cdot)$ with $\phi\left(P^{*}, \cdot\right)$, then $\phi(P, \cdot)$ and $\phi\left(P^{*}, \cdot\right)$ satisfy the Riccati-type equation (4.20). From the definition of meromorphic function $\phi$ and (4.21)-(4.24), we 
have

$$
\begin{aligned}
& \check{F}=\frac{2}{\phi(P)-\phi\left(P^{*}\right)}=\frac{\lambda^{N_{2}}}{\alpha_{0,+}} \frac{F(\lambda)}{y}, \\
& \check{G}=-\frac{\phi(P)+\phi\left(P^{*}\right)}{\phi(P)-\phi\left(P^{*}\right)}=\frac{\lambda^{N_{2}}}{\alpha_{0,+}} \frac{G(\lambda)}{y}, \\
& \check{H}=\frac{-2 \lambda \lambda^{-1} \phi(P) \phi\left(P^{*}\right)}{\phi(P)-\phi\left(P^{*}\right)}=\frac{\lambda^{N_{2}}}{\alpha_{0,+}} \frac{H(\lambda)}{y} .
\end{aligned}
$$

Moreover, as $P \rightarrow P_{\infty \pm}$, one obtains the following expansions using (3.5):

$$
\begin{gathered}
\frac{\lambda^{N_{2}}}{\alpha_{0,+}} \frac{F(\lambda)}{y} \underset{\substack{\zeta \rightarrow 0 \\
=}}{=} \mp \frac{\zeta}{\alpha_{0,+}}\left(\sum_{k=0}^{\infty} \hat{\alpha}_{k}(\underline{\lambda}) \zeta^{k}\right)\left(\sum_{l=0}^{\infty} \hat{f}_{l,+} \zeta^{l},\right. \\
\frac{\lambda^{N_{2}}}{\alpha_{0,+}} \frac{G(\lambda)}{y} \underset{\substack{\zeta \rightarrow 0 \\
=}}{=} \mp \frac{\zeta}{\alpha_{0,+}}\left(\sum_{k=0}^{\infty} \hat{\alpha}_{k}(\underline{\lambda}) \zeta^{k}\right)\left(\sum_{j=0}^{N_{2}-1} g_{N_{2}-1-j,-} \zeta^{N_{1}+j}+\sum_{j=0}^{N_{1}} f_{N_{1}-j,+} \zeta^{N_{1}-j}\right) \\
\substack{\zeta \rightarrow 0 \\
l=0} \hat{g}_{l,+} \zeta^{l}, \\
\frac{\lambda^{N_{2}}}{\alpha_{0,+}} \frac{H_{1}}{y(\lambda)} \underset{\substack{\zeta \rightarrow 0 \\
\zeta \rightarrow 0}}{=} \mp \frac{\zeta}{\alpha_{0,+}}\left(\sum_{k=0}^{\infty} \hat{\alpha}_{k}(\underline{\lambda}) \zeta^{k}\right)\left(\sum_{j=0}^{N_{2}-1} h_{N_{2}-1-j,-} \zeta^{N_{1}-j}\right) \\
\substack{\zeta \rightarrow 0} \sum_{l=0}^{\infty} \hat{h}_{l,+} \zeta^{l} .
\end{gathered}
$$

This implies (4.32) as $P \rightarrow P_{\infty \pm}$. Similarly, as $P \rightarrow P_{0, \pm}$,

$$
\begin{aligned}
& \frac{\lambda^{N_{2}}}{\alpha_{0,+}} \frac{F(\lambda)}{y} \underset{\zeta \rightarrow 0}{=} \pm \frac{1}{\alpha_{0,-}}\left(\sum_{k=0}^{\infty} \hat{\alpha}_{k}\left(\underline{\lambda}^{-1}\right) \zeta^{k}\right)\left(\sum_{j=0}^{N_{2}-1} f_{N_{2}-1-j,-} \zeta^{N_{1}+j}+\sum_{j=1}^{N_{1}} f_{N_{1}-j,+} \zeta^{N_{1}-j}\right) \\
& \underset{\zeta \rightarrow 0}{=} \pm \sum_{l=0}^{\infty} \hat{f}_{l,-} \zeta^{l}, \\
& \frac{\lambda^{N_{2}}}{\alpha_{0,+}} \frac{G(\lambda)}{y} \underset{\zeta \rightarrow 0}{= \pm \frac{1}{\alpha_{0,-}}}\left(\sum_{k=0}^{\infty} \hat{\alpha}_{k}\left(\underline{\lambda}^{-1}\right) \zeta^{k}\right)\left(\sum_{j=0}^{N_{2}-1} g_{N_{2}-j,-} \zeta^{N_{1}+j}+\sum_{j=1}^{N_{1}} g_{N_{1}-j,+} \zeta^{N_{1}-j}\right) \\
& \underset{\zeta \rightarrow 0}{=} \pm \sum_{l=0}^{\infty} \hat{g}_{l,-} \zeta^{l}, \\
& \frac{\lambda^{N_{2}}}{\alpha_{0,+}} \frac{H(\lambda)}{y} \underset{\zeta \rightarrow 0}{=} \pm \frac{1}{\alpha_{0,-}}\left(\sum_{k=0}^{\infty} \hat{\alpha}_{k}\left(\underline{\lambda}^{-1}\right) \zeta^{k}\right)\left(\sum_{j=0}^{N_{2}-1} h_{N_{2}-1-j,-} \zeta^{N_{1}+j}+\sum_{j=1}^{N_{1}} h_{N_{1}-j,+} \zeta^{N_{1}-j}\right) \\
& \underset{\zeta \rightarrow 0}{=} \pm \sum_{l=0}^{\infty} \hat{h}_{l,-} \zeta^{l} .
\end{aligned}
$$

Thus, (4.33) holds as $P \rightarrow P_{0, \pm}$.

Next, by comparing powers of $\zeta$ in the second and third terms of (4.46) and (4.49), respectively, formula (4.39) follows. Similarly, we can get formula (4.40) and formula (4.41). 
Finally, multiplying (4.14) and (4.17) and comparing coefficients of $\zeta^{k}$, one finds $\sum_{l=0}^{k} \alpha_{l}\left(\underline{\lambda}^{ \pm 1}\right) \hat{\alpha}_{k-l}\left(\underline{\lambda}^{ \pm 1}\right)=\delta_{k, 0}$, then, one computes

$$
\alpha_{0, \pm} \sum_{k=0}^{l} \alpha_{l-k}\left(\underline{\lambda}^{ \pm 1}\right) \hat{f}_{k, \pm}=\sum_{k=0}^{l} \alpha_{l-k}\left(\underline{\lambda}^{ \pm 1}\right) \sum_{s=0}^{k} \hat{\alpha}_{k-s}\left(\underline{\lambda}^{ \pm 1}\right) f_{s, \pm}=f_{l, \pm}, \quad k \in \mathbf{N}_{0}
$$

Hence (4.36) holds and (4.34) can be proved using(3.8). The proofs of expansions (4.37) and (4.38) follow precisely the same strategy and are hence omitted.

Assuming $N \in \mathbb{N}$ to be fixed and introducing

$$
\begin{aligned}
& \mathbf{S}_{k}=\left\{\underline{l}=\left(l_{1}, \ldots, l_{k}\right) \in \mathbb{N}^{k} \mid 1 \leq l_{1}<\ldots<l_{k} \leq N\right\}, \quad k=1, \ldots, N, \\
& \mathbf{I}_{k}^{(j)}=\left\{\underline{l}=\left(l_{1}, \ldots, l_{k}\right) \in \mathbf{S}_{k} \mid l_{i} \neq j, i=1, \ldots, k\right\}, \quad k=1, \ldots, N-1, \quad j=1, \ldots, N,
\end{aligned}
$$

one defines the symmetric functions

$$
\begin{aligned}
& \boldsymbol{\Psi}_{0}(\underline{\mu})=1, \quad \Psi_{k}(\underline{\mu})=(-1)^{k} \sum_{\underline{l} \in \mathbf{S}_{k}} \mu_{l_{1}} \ldots \mu_{l_{k}}, \quad k=1, \ldots, N, \\
& \mathbf{T}_{k}^{j} \underline{(\underline{\mu})}=(-1)^{k} \sum_{\underline{\underline{l} \in \mathbf{I}_{k}^{j j}}} \mu_{l_{1}} \ldots \mu_{l_{k}}, \quad k=1, \ldots, N-1, \quad j=1, \ldots, N, \\
& \mathbf{T}_{0}^{j}(\underline{\mu})=1, \quad \mathbf{T}_{N}^{j}(\underline{\mu})=0, \quad j=1, \ldots, N,
\end{aligned}
$$

where $\mu=\left(\mu_{1}, \ldots \mu_{N}\right) \in \mathbb{C}^{N}$.

Introducing

$$
\widetilde{W}(\lambda)=\prod_{j=1}^{N}\left(\lambda-\mu_{j}\right)=\sum_{l=0}^{N} \Psi_{l}(\mu) \lambda^{N-l}
$$

one infers

$$
\widetilde{W}_{\lambda}\left(\mu_{k}\right)=\prod_{\substack{j=1 \\ j \neq k}}^{N}\left(\mu_{k}-\mu_{j}\right)
$$

According to Lagrange interpolation theorem, we have the following important propositions (these propositions were proven in detail in [15], Theorem D.1, Lemma D.2, Lemma D.3).

Proposition 4.2. Suppose that $\mu_{1}, \ldots, \mu_{N}$ are $N$ distinct complex numbers. Then,

$$
\begin{aligned}
\sum_{j=1}^{N} \frac{\mu_{j}^{l-1}}{\widetilde{H}_{\lambda}\left(\mu_{j}\right)} \mathbf{T}_{k}^{j}(\underline{\mu})= & \delta_{l, N-k}-\Psi_{k+l}(\underline{\mu}) \delta_{l, N+1}, \\
& l=1, \ldots, N+1, \quad k=0, \ldots, N-1 .
\end{aligned}
$$


Proposition 4.3. Suppose that $\mu_{1}, \ldots, \mu_{N}$ are $N$ distinct complex numbers. Then,

(1) $\boldsymbol{\Psi}_{k+l}(\underline{\mu})+\mu_{j} \mathbf{T}_{k}^{j}(\underline{\mu})=\mathbf{T}_{k+1}^{j}(\underline{\mu}), \quad j=1, \ldots, N, \quad k=0, \ldots, N-1$.

(2) $\sum_{l=0}^{k} \boldsymbol{\Psi}_{k-l}(\underline{\mu}) \mu_{j}^{l}=\mathbf{T}_{k}^{j}(\underline{\mu}), \quad j=1, \ldots, N, \quad k=0, \ldots, N$.

Assume that $\mu_{j} \neq \mu_{j^{\prime}}$ for $j \neq j^{\prime}$, we introduce the $N \times N$ matrix $\mathbf{B}_{N}(\underline{\mu})$ by

$$
\mathbf{B}_{1}(\underline{\mu})=1, \quad \mathbf{B}_{N}(\underline{\mu})=\left(\frac{\mu_{k}^{j-1}}{\prod_{\substack{i=1 \\ i \neq k}}^{N}\left(\mu_{k}-\mu_{i}\right)}\right)_{j, k=1}^{N}
$$

where $\underline{\mu}=\left(\mu_{1}, \ldots \mu_{N}\right) \in \mathbb{C}^{N}$.

Proposition 4.4. Suppose that $\mu_{1}, \ldots, \mu_{N}$ are $N$ distinct complex numbers. Then,

$$
\mathbf{B}_{N}(\underline{\mu})^{-1}=\left(\mathbf{T}_{N-k}^{j}(\underline{\mu})\right)_{j, k=1}^{N} .
$$

From (3.9), we have

$$
\begin{aligned}
& \lambda^{N_{2}} F(n)=\alpha_{0,+} \varepsilon u \prod_{l=1}^{N}\left(\lambda-\mu_{j}\right)=\alpha_{0,+} \varepsilon u \sum_{l=0}^{N} \Psi_{l}(\underline{\mu}) \lambda^{N-l}, \\
& \lambda^{N_{2}} H(n)=\alpha_{0,+} \varepsilon v^{-} \prod_{l=1}^{N}\left(\lambda-v_{j}\right)=\alpha_{0,+} \varepsilon v^{-} \sum_{l=0}^{N} \Psi_{l}(\underline{v}) \lambda^{N-l} .
\end{aligned}
$$

Then

$$
\begin{aligned}
& f_{l,+}=\alpha_{0,+} \varepsilon u \Psi_{l}(\underline{\mu}), \quad 1 \leq l \leq N_{1}, \quad f_{l,-}=\alpha_{0,+} \varepsilon u \Psi_{N-l}(\underline{\mu}), \quad 0 \leq l \leq N_{2}-1, \\
& h_{l,+}=\alpha_{0,+} \varepsilon v^{-} \Psi_{l}(\underline{v}), \quad 1 \leq l \leq N_{1}, \quad h_{l,-}=\alpha_{0,+} \varepsilon v^{-} \boldsymbol{\Psi}_{N-l}(\underline{v}), \quad 0 \leq l \leq N_{2}-1 .
\end{aligned}
$$

Theorem 4.5. (Straightening out of the continuous flow)

$$
\partial_{t_{\underline{m}}} \underline{\rho}^{(1)}\left(n, t_{\underline{m}}\right)=\underline{\mathbf{Y}}^{(\underline{m})}, \quad \partial_{t_{\underline{m}}} \underline{\rho}^{(2)}\left(n, t_{\underline{m}}\right)=-\underline{\mathbf{Y}}^{(\underline{m})},
$$

where

$$
\begin{gathered}
\underline{\mathbf{Y}}^{(\underline{m})}=\sum_{s=1}^{m} \widetilde{\alpha}_{m-s,+} \sum_{q=0}^{s-1} \underline{C}_{N-q} \hat{\alpha}_{s-1-q}(\underline{\lambda})-\left(\prod_{j=1}^{2 N+2} \lambda_{j}\right)^{-\frac{1}{2}} \sum_{s=1}^{m} \widetilde{\alpha}_{m-s,+} \sum_{q=0}^{s-1} \underline{C}_{s-q} \hat{\alpha}_{q}\left(\underline{\lambda}^{-1}\right), \\
\underline{\rho}\left(n, t_{\underline{m}}\right)=\left(\rho_{1}\left(n, t_{\underline{m}}\right), \ldots, \rho_{N}\left(n, t_{\underline{m}}\right)\right), \underline{C}_{r}=\left(C_{1 r}, \ldots, C_{N r}\right), 1 \leq r \leq N .
\end{gathered}
$$


Proof. From (3.8), we arrive at

$$
f_{k,+}(n)=\sum_{j=0}^{k} \alpha_{j,+} \hat{f}_{k-j,+}(n), \quad f_{k,-}(n)=\sum_{j=0}^{k} \alpha_{j,-} \hat{f}_{k-j,-}(n),
$$

from (4.39) we know that

$$
\begin{gathered}
\hat{f}_{k,+}(n)=\frac{1}{\alpha_{0,+}} \sum_{l=0}^{k-1} \hat{\alpha}_{l-k}(\underline{\lambda}) f_{k-l,+}(n)=\varepsilon u \sum_{l=0}^{k-1} \hat{\alpha}_{k-l}(\underline{\boldsymbol{\lambda}}) \boldsymbol{\Psi}_{k-l-1}(\underline{\mu})=\varepsilon u \sum_{l=0}^{k-1} \hat{\alpha}_{k-l-1}(\underline{\boldsymbol{\lambda}}) \boldsymbol{\Psi}_{l}(\underline{\mu}), \\
\hat{f}_{k,-}(n)=\frac{1}{\alpha_{0,-}} \sum_{l=0}^{k-1} \hat{\alpha}_{l-k}\left(\underline{\lambda}^{-1}\right) f_{k,-}(n)=\frac{\alpha_{0,+}}{\alpha_{0,-}} \varepsilon u \sum_{l=0}^{k} \hat{\alpha}_{l-k}\left(\underline{\lambda}^{-1}\right) \Psi_{N-k}(\underline{\mu}) .
\end{gathered}
$$

By definition, we have

$$
\begin{gathered}
\widehat{F}_{l,+}=\sum_{k=1}^{l} \hat{f}_{k,+} \lambda^{l-k}=\varepsilon u \sum_{k=1}^{l} \sum_{s=0}^{k-1} \hat{\alpha}_{k-1-s}(\underline{\lambda}) \Psi_{s}(\underline{\mu}) \lambda^{l-k} \\
=\varepsilon u \sum_{k=0}^{l-1} \hat{\alpha}_{l-1-k}(\underline{\lambda}) \sum_{s=0}^{k} \Psi_{s}(\underline{\mu}) \lambda^{k-s}, \\
\widehat{F}_{l,-}=\sum_{k=0}^{l-1} \hat{f}_{k,-} \lambda^{k-l}=\varepsilon u \frac{\alpha_{+, 0}}{\alpha_{-, 0}} \sum_{k=0}^{l-1} \sum_{s=0}^{k} \hat{\gamma}_{k-s} \Psi_{N-s}(\underline{\mu}) \lambda^{k-l} \\
=-\varepsilon u \frac{\alpha_{0,+}}{\alpha_{0,-}} \sum_{k=0}^{l-1} \hat{\alpha}_{k}\left(\underline{\lambda}^{-1}\right) \sum_{s=N-l+k+1}^{N} \Psi_{s}(\underline{\mu}) \lambda^{N-l+k-s}
\end{gathered}
$$

Hence,

$$
\begin{gathered}
\widehat{F}_{l,+}\left(\mu_{k}\right)=\varepsilon u \sum_{j=0}^{l-1} \hat{\alpha}_{k-1-j}(\underline{\boldsymbol{\lambda}}) \sum_{s=0}^{j} \Psi_{s}(\underline{\mu}) \mu_{k}^{j-s}=\varepsilon u \sum_{j=0}^{l-1} \hat{\alpha}_{l-1-j}(\underline{\lambda}) \mathbf{T}_{j}^{(k)}(\underline{\mu}), \quad 1 \leq l \leq m, \\
\widehat{F}_{l,-}\left(\mu_{k}\right)=-\varepsilon u \frac{\alpha_{0,+}}{\alpha_{0,-}} \sum_{j=0}^{l-1} \hat{\alpha}_{j}\left(\underline{\boldsymbol{\lambda}}^{-1}\right) \sum_{s=0}^{N-l+j} \boldsymbol{\Psi}_{s}(\underline{\mu}) \mu_{j}^{N-l+j-s}=-\varepsilon u \frac{\alpha_{0,+}}{\alpha_{0,-}} \sum_{j=0}^{l-1} \hat{\alpha}_{j}\left(\underline{\boldsymbol{\lambda}}^{-1}\right) \mathbf{T}_{N-l+k}^{(j)}(\underline{\mu}), \\
0 \leq l \leq m-1,
\end{gathered}
$$

Then

$$
\begin{aligned}
& \widetilde{F}_{\underline{m}}\left(\mu_{k}\right)=\sum_{s=1}^{m} \widetilde{\alpha}_{m-s,+} \widehat{F}_{s,+}\left(\mu_{k}\right)+\sum_{s=1}^{m} \widetilde{\alpha}_{m-s,-} \widehat{F}_{s,-}\left(\mu_{k}\right) \\
& =\varepsilon u \sum_{s=1}^{m} \widetilde{\alpha}_{m-s,+} \sum_{j=0}^{s-1} \hat{\alpha}_{s-1-j}(\underline{\lambda}) \mathbf{T}_{j}^{(k)}(\underline{\mu})-\varepsilon u \frac{\alpha_{0,+}}{\alpha_{0,-}} \sum_{s=1}^{m} \widetilde{\alpha}_{m-s,-} \sum_{j=0}^{s-1} \hat{\alpha}_{j}\left(\underline{\lambda}^{-1}\right) \mathbf{T}_{N-s+j}^{(k)}(\underline{\mu}) \\
& =\varepsilon u \sum_{s=1}^{m} \widetilde{\alpha}_{m-s,+} \sum_{j=0}^{s-1} \hat{\alpha}_{s-1-j}(\underline{\lambda}) \mathbf{B}_{N}(\underline{\mu})_{k, N-j}^{-1}-\varepsilon u \frac{\alpha_{0,+}}{\alpha_{0,-}} \sum_{s=1}^{m} \widetilde{\alpha}_{m-s,-} \sum_{j=0}^{s-1} \hat{\alpha}_{j}\left(\underline{\lambda}^{-1}\right) \mathbf{B}_{N}(\underline{\mu})_{k, s-j}^{-1} .
\end{aligned}
$$


From (4.13) and (4.66), we obtain

$$
\begin{aligned}
& \partial_{t_{\underline{m}}} \rho_{j}^{(1)}\left(n, t_{\underline{m}}\right)=\sum_{l=1}^{N} \sum_{k=1}^{N} C_{j l} \frac{\mu_{k}^{l-1} \mu_{k, t_{\underline{m}}}}{y\left(\hat{\mu}_{k}\right)}=\sum_{l=1}^{N} \sum_{k=1}^{N} \frac{C_{j l} \mu_{k}^{l-1} \widetilde{F}_{\underline{m}}\left(\mu_{k}\right)}{\varepsilon u \prod_{\substack{i=1 \\
i \neq k}}^{N}\left(\mu_{k}-\mu_{i}\right)}=\sum_{j=1}^{N} \sum_{l=1}^{N} C_{j l} \mathbf{B}_{N}(\underline{\mu})_{l, j} \frac{\widetilde{F}_{\underline{m}}\left(\mu_{j}\right)}{\varepsilon u} \\
& =\sum_{s=1}^{m} \widetilde{\alpha}_{m-s,+} \sum_{q=0}^{s-1} C_{j, N-q} \hat{\alpha}_{s-1-q}(\underline{\lambda})-\left(\prod_{j=1}^{2 N+2} \lambda_{j}\right)^{-\frac{1}{2}} \sum_{s=1}^{m} \widetilde{\alpha}_{m-s,+} \sum_{q=0}^{s-1} C_{j, s-q} \hat{\alpha}_{q}\left(\underline{\lambda}^{-1}\right)=\mathbf{Y}_{j}^{(\underline{m})} .
\end{aligned}
$$

In a similar way, we can prove the second expression of (4.58).

\section{Quasi-periodic solutions}

In this section, we shall construct quasi-periodic solutions of the discrete self-dual network hierarchy. We recall that we have defined the meromorphic function $\phi\left(\cdot, n, t_{\underline{m}}\right)$ on $\mathscr{K}_{N}$ in (4.5), then the time-dependent Baker-Akhiezer vector is defined in terms of $\phi\left(\cdot, n, t_{\underline{m}}\right)$ by

$$
\begin{aligned}
& \Psi\left(P, n, n_{0}, t_{\underline{m}}, t_{0, \underline{m}}\right)=\left(\begin{array}{l}
\psi_{1}\left(P, n, n_{0}, t_{\underline{m}}, t_{0, \underline{m}}\right) \\
\psi_{2}\left(P, n, n_{0}, \underline{t_{\underline{m}}}, t_{0, \underline{m}}\right)
\end{array}\right), \\
& \psi_{1}\left(P, n, n_{0}, t_{\underline{m}}, t_{0, \underline{m}}\right)=\quad \exp \left(\int_{t_{0, \underline{m}}}^{t_{\underline{m}}} d s\left(\widetilde{G}\left(\lambda, n_{0}, s\right)+\widetilde{F}\left(\lambda, n_{0}, s\right) \phi\left(P, n_{0}, s\right)\right)\right. \\
& \times\left\{\begin{array}{lr}
\prod_{n^{\prime}=n_{0}}^{n-1}\left[\lambda+u v+\left(\varepsilon u+\lambda^{-1} v\right) \phi\left(P, n^{\prime}, t_{\underline{m}}\right)\right], & n \geq n_{0}+1, \\
1, & n=n_{0} \\
\prod_{n^{\prime}=n}^{n_{0}-1}\left[\lambda+u v+\left(\varepsilon u+\lambda^{-1} v\right) \phi\left(P, n^{\prime}, t_{\underline{m}}\right)\right]^{-1}, & n \leq n_{0}-1
\end{array}\right. \\
& \psi_{2}\left(P, n, n_{0}, t_{\underline{m}}, t_{0, \underline{m}}\right)=\exp \left(\int_{t_{0, \underline{m}}}^{t_{\underline{m}}} d s\left(\widetilde{G}\left(\lambda, n_{0}, s\right)+\widetilde{F}\left(\lambda, n_{0}, s\right) \phi\left(P, n_{0}, s\right)\right)\right. \\
& \times \phi\left(P, n_{0}, t_{\underline{m}}\right)\left\{\begin{array}{lc}
\prod_{n^{\prime}=n_{0}}^{n-1}\left[\lambda^{-1}+u v+(u+\lambda \varepsilon v) \phi\left(P, n^{\prime}, t_{\underline{m}}\right)^{-1}\right], & n \geq n_{0}+1, \\
1, & n=n_{0} \\
\prod_{n^{\prime}=n}^{n_{0}-1}\left[\lambda^{-1}+u v+(u+\lambda \varepsilon v) \phi\left(P, n^{\prime}, t_{\underline{m}}\right)^{-1}\right]^{-1}, & n \leq n_{0}-1,
\end{array}\right.
\end{aligned}
$$


where $u=u\left(n^{\prime}, t_{\underline{m}}\right), v=v\left(n^{\prime}, t_{\underline{m}}\right), P=(\lambda, y) \in \mathscr{K}_{N} \backslash\left\{P_{\infty+}, P_{\infty-}, P_{0,+}, P_{0,-}\right\},\left(n, t_{\underline{m}}\right) \in \mathbb{Z} \times \mathbb{R}$. One observes that

$$
\begin{array}{r}
\psi_{1}\left(P, n, n_{0}, t_{\underline{m}}, \tilde{t}_{\underline{m}}\right)=\psi_{1}\left(P, n_{0,}, n_{0}, t_{\underline{m}}, \tilde{t}_{\underline{m}}\right) \psi_{1}\left(P, n, n_{0}, t_{\underline{m}}, t_{\underline{m}}\right), \\
P=(\lambda, y) \in \mathscr{K}_{N} \backslash\left\{P_{\infty+}, P_{\infty-}, P_{0,+}, P_{0,-}\right\}, \quad\left(n, n_{0}, t_{\underline{m}}, t_{0, \underline{m}}\right) \in \mathbb{Z}^{2} \times \mathbb{R}^{2} .
\end{array}
$$

Lemma 5.1. Suppose that $u\left(n, t_{\underline{m}}\right), v\left(n, t_{\underline{m}}\right)$ satisfy the hierarchy of discrete self-dual network equations (2.29). Let $\lambda_{j} \in \mathbb{C} \backslash\{0\},(1 \leq j \leq 2 N+2)$, and $P=(\lambda, y) \in \mathscr{K}_{N} \backslash\left\{P_{\infty+}, P_{\infty-}, P_{0,+}, P_{0,-}\right\}$, $\left(n, t_{\underline{m}}\right) \in \mathbb{Z} \times \mathbb{R}$. Then

$$
\begin{array}{cc}
\phi(P) \underset{\zeta \rightarrow 0}{=}\left\{\begin{array}{lc}
-\frac{1}{\varepsilon u} \zeta^{-1}+\frac{v}{u^{2}} \gamma^{2}+O(\zeta), & \text { as } P \rightarrow P_{\infty+}, \\
\varepsilon v^{-}+u^{-}\left(\pi^{-}\right)^{2} \zeta+O\left(\zeta^{2}\right), & \text { as } P \rightarrow P_{\infty-} ;
\end{array} \quad \text { as } P \rightarrow P_{0,+},\right. \\
\phi(P)= \begin{cases}\frac{1}{v^{-}}-\frac{\varepsilon u^{-}}{\left(v^{-}\right)^{2}}\left(\pi^{-}\right)^{2} \zeta+O\left(\zeta^{2}\right), & \zeta=\lambda, \\
-u \zeta-\varepsilon v \gamma^{2} \zeta^{2}+O\left(\zeta^{3}\right), & \text { as } P \rightarrow P_{0,-} .\end{cases}
\end{array}
$$

Proof. Introducing the local coordinate $\zeta=\lambda^{-1}$ near $P_{\infty \pm}$ and $\zeta=\lambda$ near $P_{0, \pm}$, from (3.5), (3.9) and (4.5), we have

$$
\begin{aligned}
& G \underset{\zeta \rightarrow 0}{=} \begin{cases}\zeta^{-N_{1}}\left(g_{0,+}+g_{1,+} \zeta+g_{2,+} \zeta^{2}+g_{3,+} \zeta^{3}+O\left(\zeta^{4}\right)\right), & \text { as } P \rightarrow P_{\infty \pm} \\
\zeta^{-N_{2}}\left(g_{0,-}+g_{1,-} \zeta+O\left(\zeta^{2}\right)\right), & \text { as } P \rightarrow P_{0, \pm}\end{cases} \\
& F^{-1}=\frac{\lambda^{N_{2}}}{\alpha_{0,+} \varepsilon u} \prod_{j=1}^{N}\left(\lambda-\mu_{j}\right)^{-1} \\
& =\left\{\begin{array}{l}
\frac{\zeta^{N_{1}-1}}{\alpha_{0,+} \varepsilon u} \prod_{j=1}^{N}\left(1-\mu_{j} \zeta\right)^{-1} \underset{\zeta \rightarrow 0}{=} \frac{\zeta^{N_{1}-1}}{\alpha_{0,+} \varepsilon u}\left(1+\sum_{j=1}^{N} \mu_{j} \zeta+O\left(\zeta^{2}\right)\right), \quad \text { as } P \rightarrow P_{\infty \pm}, \\
\frac{(-1)^{N} \zeta^{N_{2}}}{\alpha_{0,+} \varepsilon \prod_{j=1}^{N} \mu_{j}} \prod_{j=1}^{N}\left(1-\mu_{j}^{-1} \zeta\right)^{-1} \underset{\zeta \rightarrow 0}{=} \frac{1}{\alpha_{0,-} v^{-}}\left(1+\sum_{j=1}^{N} \mu_{j}^{-1} \zeta+O\left(\zeta^{2}\right)\right), \text { as } P \rightarrow P_{0, \pm},
\end{array}\right.
\end{aligned}
$$

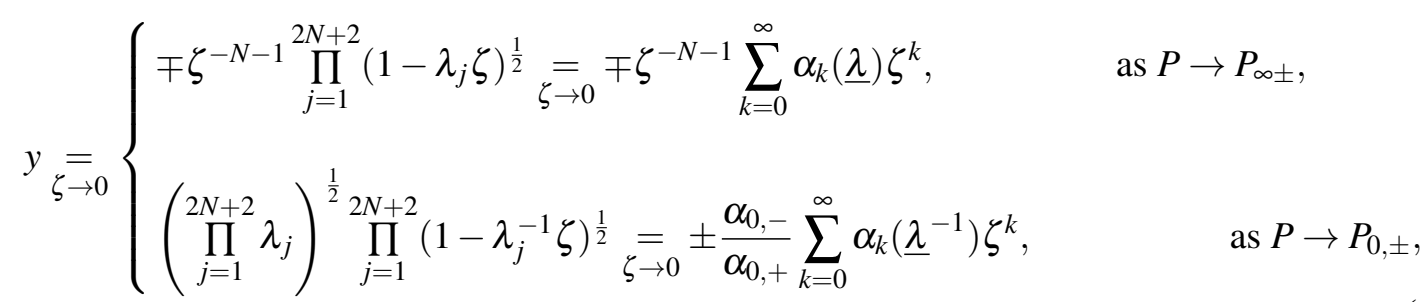


where $\underline{\lambda}=\left(\lambda_{1}, \ldots, \lambda_{2 N+2}\right), \underline{\lambda}^{-1}=\left(\lambda_{1}^{-1}, \ldots, \lambda_{2 N+2}^{-1}\right)$,

$$
\alpha_{0}\left(\underline{\lambda}^{ \pm 1}\right)=1, \quad \alpha_{k}\left(\underline{\lambda}^{ \pm 1}\right)=\sum_{\substack{j_{1} \ldots, j_{2 N+2}=0 \\ j_{1}+\ldots+j_{2 N+2}=k}}^{k} \frac{\left(2 j_{1}\right) ! \ldots\left(2 j_{2 N+2}\right) ! \lambda_{1}^{ \pm j_{1}} \ldots \lambda_{2 N+2}^{ \pm j_{2 N+2}}}{2^{2 k}\left(j_{1} !\right)^{2} \ldots\left(j_{2 N+2} !\right)^{2}\left(2 j_{1}-1\right) \ldots\left(2 j_{2 N+2}-1\right)}, \quad k \in \mathbb{N} .
$$

Then according to the definition of $\phi$ in (4.5), we finally obtain that

$$
\begin{aligned}
& \phi\left(P, n, t_{\underline{m}}\right)=\left(\frac{1}{2} \alpha_{0,+} \lambda^{-N_{2}} y-G\right) \times F^{-1} \\
& =\left\{\begin{array}{l}
{\left[\mp \frac{\alpha_{0,+}}{2}\left(1+\alpha_{1}(\underline{\lambda}) \zeta+\alpha_{2}(\underline{\lambda}) \zeta^{2}+\alpha_{3}(\underline{\lambda}) \zeta^{3}+O\left(\zeta^{4}\right)\right)-\left(g_{0,+}+g_{1,+} \zeta+g_{2,+} \zeta^{2}+g_{3} \zeta^{3}+O\left(\zeta^{4}\right)\right)\right]} \\
\times\left(1+\sum_{j=1}^{N} \mu_{j} \zeta+O\left(\zeta^{2}\right)\right) \frac{1}{\alpha_{0,+} \varepsilon u} \zeta^{-1}, \quad \text { as } P \rightarrow P_{\infty \pm}, \\
{\left[ \pm \frac{\alpha_{0,-}}{2}\left(1+\alpha_{1}\left(\underline{\lambda}^{-1}\right) \zeta+\alpha_{2}\left(\underline{\lambda}^{-1}\right) \zeta^{2}+O\left(\zeta^{3}\right)\right)-\left(g_{0,-}+g_{1,-} \zeta+O\left(\zeta^{2}\right)\right)\right]} \\
\times \frac{1}{\alpha_{0,-} v^{-}}(1+O(\zeta)),
\end{array}\right.
\end{aligned}
$$

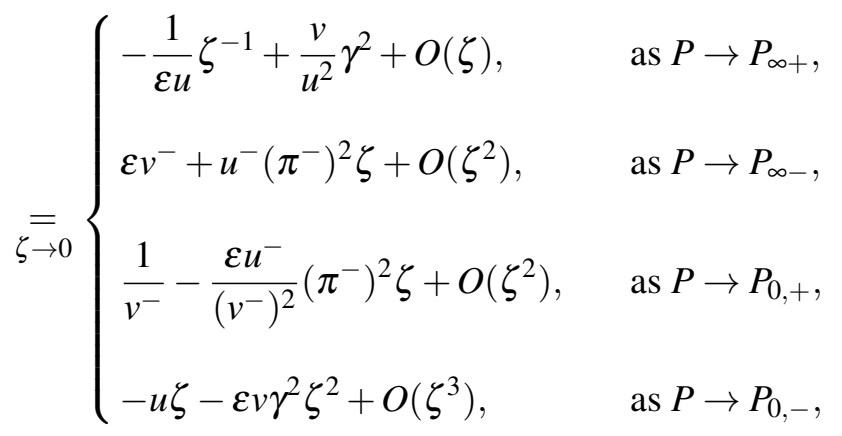

which proves (5.5) and (5.6).

Theorem 5.2. Assume Hypothesis 2.1 and suppose that (2.29), (3.2) hold. Moreover, let $P=$ $(\lambda, y) \in \mathscr{K}_{N} \backslash\left\{P_{\infty+}, P_{\infty-}, P_{0,+}, P_{0,-}\right\},\left(n, n_{0}, t_{\underline{m}}, t_{0, \underline{m}}\right) \in \mathbb{Z}^{2} \times \mathbb{R}^{2}$. Then the component $\psi_{1}$ of the BakerAkhiezer vector $\Psi$ has the asymptotic behavior

$$
\begin{aligned}
& \left.\psi_{1}\left(P, n, n_{0}, t_{\underline{t}}, t_{0, \underline{\underline{m}}}\right) \underset{\zeta \rightarrow 0}{=} \exp \left(\mp \frac{1}{2}\left(t_{\underline{m}}-t_{0, \underline{m}}\right) \sum_{s=0}^{m} \tilde{\alpha}_{m-s,+} \zeta^{-s}\right)\right)(1+O(\zeta)) \\
& \times\left\{\begin{array}{cc}
\frac{u\left(n, t_{\underline{m}}\right)}{u\left(n_{0}, t_{0, \underline{\underline{m}}}\right)} \zeta^{n-n_{0}} \Gamma\left(n, n_{0}, t_{\underline{m}}\right) \exp \left(\int_{t_{0, \underline{\underline{m}}}}^{t_{\underline{m}}} \tilde{g}_{m,+}\left(n_{0}, s\right)\right) d s, & \text { as } P \rightarrow P_{\infty+}, \\
\zeta^{\left(n_{0}-n\right)} \exp \left(\int_{t_{0, \underline{m}}}^{t_{\underline{m}}^{\underline{\underline{m}}}} \tilde{g}_{m,-}\left(n_{0}, s\right) d s\right), & \text { as } P \rightarrow P_{\infty-},
\end{array} \quad \zeta=\lambda^{-1},\right.
\end{aligned}
$$




$$
\begin{aligned}
& \left.\psi_{1}\left(P, n, n_{0}, t_{\underline{m}}, t_{0, \underline{m}}\right) \underset{\zeta \rightarrow 0}{=} \exp \left( \pm \frac{1}{2}\left(t_{\underline{m}}-t_{0, \underline{m}}\right) \sum_{s=0}^{m} \tilde{\alpha}_{m-s,+} \zeta^{-s}\right)\right)(1+O(\zeta)) \\
& \quad \times\left\{\begin{array}{lr}
\zeta^{n_{0}-n} \frac{v^{-}\left(n, t_{\underline{m}}\right)}{v^{-}\left(n_{0}, t_{0, \underline{m}}\right)} \exp \left(\int_{t_{0, \underline{m}}}^{t_{\underline{m}}} \tilde{g}_{m,-}\left(n_{0}, s\right)\right) d s, & \text { as } P \rightarrow P_{0,+}, \\
\zeta^{n-n_{0}} \Gamma\left(n, n_{0}, t_{\underline{m}}\right) \exp \left(\int_{t_{0, \underline{m}}}^{t_{\underline{m}}^{\underline{\underline{g}}}} \tilde{g}_{m,+}\left(n_{0}, s\right)\right) d s, & \text { as } P \rightarrow P_{0,-},
\end{array} \quad \zeta=\lambda,\right.
\end{aligned}
$$

where

$$
\Gamma\left(n, n_{0}, t_{\underline{m}}\right)=\left\{\begin{array}{lc}
\prod_{n^{\prime}=n_{0}}^{n-1} \gamma^{2}\left(n^{\prime}, t_{\underline{m}}\right) \pi^{2}\left(n^{\prime}, t_{\underline{m}}\right), & n \geq n_{0}+1 \\
1, & n=n_{0} \\
\prod_{n^{\prime}=n}^{n_{0}-1}\left[\gamma^{2}\left(n^{\prime}, t_{\underline{m}}\right) \pi^{2}\left(n^{\prime}, t_{\underline{m}}\right)\right]^{-1}, & n \leq n_{0}-1
\end{array}\right.
$$

The divisors $\left(\psi_{1}\left(\cdot n, n_{0}, t_{\underline{m}}, t_{0, \underline{m}}\right)\right)$, is given by

$$
\left(\psi_{1}\left(\cdot, n, n_{0}, t_{\underline{m}}, t_{0, \underline{m}}\right)\right)=D_{\hat{\mu}\left(n, \underline{t_{\underline{m}}}\right)}-D_{\hat{\mu}\left(n_{0}, t_{0, \underline{m}}\right)}+\left(n-n_{0}\right)\left(D_{P_{0,-}}+D_{P_{\infty+}}-D_{P_{0,+}}-D_{P_{\infty-}}\right) .
$$

Proof. First, we compute the divisor $\left(\psi_{1}\left(\cdot, n, n_{0}, t_{\underline{m}}, t_{0, \underline{m}}\right)\right)$ of $\psi_{1}\left(\cdot, n, t_{\underline{m}}, t_{0, \underline{m}}\right)$. according to (5.4), we need to calculate the divisors $\left(\psi_{1}\left(\cdot, n, n_{0}, t_{\underline{m}}, t_{\underline{m}}\right)\right),\left(\psi_{1}\left(\cdot, n_{0}, n_{0}, t_{\underline{m}}, t_{0, \underline{m}}\right)\right)$, respectively. In order to calculate the divisor $\left(\psi_{1}\left(\cdot, n, n_{0}, t_{\underline{m}}, t_{\underline{m}}\right)\right)$, by (5.2) it suffices to compute the divisor of $\lambda+u v+\left(\lambda^{-1} v+\varepsilon u\right) \phi(P)$. First of all we note that

$$
\lambda+u v+\left(\lambda^{-1} v+\varepsilon u\right) \phi(P)= \begin{cases}\frac{u^{+}}{u}(\gamma \pi)^{2} \zeta+O\left(\zeta^{2}\right), & P \rightarrow P_{\infty+}, \\ \zeta^{-1}+u v+u v^{-}+O(\zeta), & P \rightarrow P_{\infty-}, \quad \zeta=\frac{1}{\lambda}, \\ \frac{v}{v^{-}} \zeta^{-1}+O(1), & P \rightarrow P_{0,+}, \quad \\ (\gamma \pi)^{2} \zeta+O\left(\zeta^{2}\right), & P \rightarrow P_{0,-}, \quad \zeta=\lambda .\end{cases}
$$

which proves (5.11),(5.12) for $t_{0, m}=t_{m}$. Moreover, the poles of the function $\lambda+u v+\left(\lambda^{-1} v+\right.$ $\varepsilon u) \phi(P)$ in $\mathscr{K}_{N} \backslash\left\{P_{\infty \pm}, P_{0, \pm}\right\}$ coincide with the ones of $\phi(P)$, and so it remains to compute the missing $N$ zeros in $\mathscr{K}_{N} \backslash\left\{P_{\infty \pm}, P_{0, \pm}\right\}$. Using (3.3), (3.14), (4.5) and $y\left(\hat{\mu}_{j}\right)=\left(-2 / \alpha_{0,+}\right) \mu_{j}^{N_{2}} G\left(\mu_{j}\right)$ (cf.(4.2)), one computes

$$
\begin{aligned}
\lambda & +u v+\left(\varepsilon u+\lambda^{-1} v\right) \phi(P)=\lambda+u v+\left(\varepsilon u+\lambda^{-1} v\right) \frac{\left(\alpha_{0,+} / 2\right) \lambda^{-N_{2}} y-G}{F} \\
& =\frac{\left(\lambda^{-1}+u v\right) F^{+}+\left(\varepsilon u+\lambda^{-1} v\right)\left(\left(\alpha_{0,+} / 2\right) \lambda^{-N_{2}} y+G^{+}\right)}{F} \\
& =\left(\lambda^{-1}+u v\right) \frac{F^{+}}{F}+\left(\varepsilon u+\lambda^{-1} v\right) \frac{\left(\alpha_{0,+} / 2\right)^{2} \lambda^{-2 N_{2}} y^{2}-\left(G^{+}\right)^{2}}{F\left(\left(\alpha_{0,+} / 2\right) \lambda^{-N_{2}} y-G^{+}\right)} \\
& =\frac{F^{+}}{F}\left(\lambda^{-1}+u v+\frac{(\lambda \varepsilon u+v) H^{+}}{\left(\alpha_{+, 0} / 2\right) \lambda^{-N_{2}} y-G^{+}}\right)_{\substack{P \rightarrow \hat{\mu}_{j} \\
=}} \frac{F^{+}(P)}{F(P)} O(1) .
\end{aligned}
$$


Hence the sought after zeros are at $\hat{\mu}_{j}, j=1, \ldots, N$ (with the possibility that a zero at $\hat{\mu}_{j}^{+}$is cancelled by a pole at $\hat{\mu}_{j}$ ). Thus,

$$
\left(\psi_{1}\left(\cdot, n, n_{0}, t_{\underline{m}}, t_{\underline{m}}\right)\right)=D_{\underline{\hat{\mu}}\left(n, t_{\underline{m}}\right)}-D_{\underline{\hat{\mu}}\left(n_{0}, t_{\underline{m}}\right)}+\left(n-n_{0}\right)\left(D_{P_{\infty+}}+D_{P_{0,-}}-D_{P_{\infty-}}-D_{P_{0,+}}\right) .
$$

on the other hand, $\psi_{1}\left(P, n_{0}, n_{0}, t_{\underline{m}}, t_{0, \underline{m}}\right)$ has zero and poles only at poles of $\phi\left(P, n_{0}, s\right), s \in$ $\left[t_{0, \underline{m}}, t_{\underline{m}}\right]\left(\right.$ resp., $\left.s \in\left[t_{\underline{m}}, t_{0, \underline{m}}\right]\right)$. In the following we temporarily restrict $t_{0, \underline{m}}$ and $t_{\underline{m}}$ to a sufficiently small nonempty interval $I \subseteq \mathbb{R}$ and pick $n_{0} \in \mathbb{Z}$ such that for all $s \in I, \mu_{j}\left(n_{0}, s\right) \neq \mu_{k}\left(n_{0}, s\right)$ for all $j \neq k, j, k=1, \ldots, N$. Using (3.4), (3.14), (4.5) and $y\left(\hat{\mu}_{j}\right)=\left(-2 / \alpha_{0+}\right) \mu_{j}^{N_{2}} G\left(\mu_{j}\right)$ (cf.(4.2)), one computes

$$
\widetilde{G}_{\underline{m}}\left(\lambda, n_{0}, s\right)+\widetilde{F}_{\underline{m}}\left(\lambda, n_{0}, s\right) \phi\left(P, n_{0}, s\right) \underset{P \rightarrow \hat{\mu}_{j}\left(n_{0}, s\right)}{=} \partial_{s} \ln \left(\lambda-\mu_{j}\left(n_{0}, s\right)\right)+O(1) .
$$

Restricting P to a sufficiently small neighborhood $\mathscr{U}_{j}\left(n_{0}, s\right)$ of $\left\{\hat{\mu}_{j}\left(n_{0}\right) \in \mathscr{K}_{N} \mid s \in\left[t_{0, \underline{m}}, t_{\underline{m}}\right] \subseteq I\right\}$ such that $\hat{\mu}_{k}\left(n_{0}, s\right) \notin \mathscr{U}_{j}\left(n_{0}\right)$ for all $s \in\left[t_{0, \underline{m}}, t_{\underline{m}}\right] \subseteq I$ and all $k \in\{1, \ldots, N\} \backslash\{j\}$, (5.17) implies

$$
\psi_{1}\left(P, n_{0}, n_{0}, t_{\underline{m}}, t_{0, \underline{m}}\right)= \begin{cases}\left(\lambda-\mu_{j}\left(n_{0}, t_{\underline{m}}\right)\right) O(1) & \text { as } P \rightarrow \hat{\mu}_{j}\left(n_{0}, t_{\underline{m}}\right) \neq \hat{\mu}_{j}\left(n_{0}, t_{0, \underline{m}}\right), \\ O(1) & \text { as } P \rightarrow \hat{\mu}_{j}\left(n_{0}, t_{\underline{m}}\right)=\hat{\mu}_{j}\left(n_{0}, t_{0, \underline{m}}\right), \\ \left(\lambda-\mu_{j}\left(n_{0}, t_{0, \underline{m}}\right)\right)^{-1} O(1) & \text { as } P \rightarrow \hat{\mu}_{j}\left(n_{0}, t_{0, \underline{m}}\right) \neq \hat{\mu}_{j}\left(n_{0}, t_{\underline{m}}\right),\end{cases}
$$

with $P=(\lambda, y) \in \mathscr{K}_{N}$, and $O(1) \neq 0$. Therefore,

$$
\left(\psi_{1}\left(\cdot, n_{0}, n_{0}, t_{\underline{m}}, t_{0, \underline{m}}\right)\right)=D_{\underline{\hat{\mu}}\left(n_{0}, t_{\underline{m}}\right)}-D_{\underline{\hat{\mu}}\left(n_{0}, t_{0, \underline{m}}\right)},
$$

according to (5.4), (5.14) holds.

In order to prove (5.11) and (5.12), it remains to investigate

$$
\psi_{1}\left(P, n_{0}, n_{0}, t_{\underline{m}}, t_{0, \underline{m}}\right)=\exp \left(\int_{t_{0, \underline{m}}}^{t_{\underline{m}}} d s\left(\widetilde{G}\left(\lambda, n_{0}, s\right)+\widetilde{F}\left(\lambda, n_{0}, s\right) \phi\left(P, n_{0}, s\right)\right) .\right.
$$

The asymptotic expansion of the integrand is derived using Theorem 4.1, focusing on the homogeneous coefficients first, one computes as $P \rightarrow P_{\infty \pm}$.

$$
\begin{aligned}
\widehat{G}_{s,+}+\widehat{F}_{s,+} \phi(P) & =\widehat{G}_{s,+}+\widehat{F}_{s,+} \frac{\frac{1}{2} \alpha_{0,+} \lambda^{-N_{2}} y-G}{F}=\widehat{G}_{s,+}-\widehat{F}_{s,+}\left(\frac{2 \lambda^{N_{2}} G}{\alpha_{0,+} y}-1\right)\left(\frac{2 \lambda^{N_{2}} F}{\alpha_{0,+} y}\right)^{-1} \\
\underset{\zeta \rightarrow 0}{=} \mp \frac{1}{2} \zeta^{-s}+\frac{\hat{g}_{0,+} \pm \frac{1}{2}}{\hat{f}_{1,+}} \hat{f}_{s+1,+}+O(\zeta), \quad P \rightarrow P_{\infty \pm}, & \zeta=\lambda^{-1} .
\end{aligned}
$$

Since $\widetilde{G}_{\underline{m}} \underset{\zeta \rightarrow 0}{=} \sum_{s=0}^{m} \tilde{\alpha}_{m-s,+} \widehat{G}_{s,+}+\tilde{g}_{m,-}+O(\zeta), \quad \widetilde{F}_{\underline{m}} \underset{\zeta \rightarrow 0}{=} \sum_{s=0}^{m} \tilde{\alpha}_{m-s,+} \widehat{F}_{s,+}+O(\zeta), \quad$ one infers from (3.6) and (5.5)

$$
\widetilde{G}_{\underline{m}}+\widetilde{F}_{\underline{m}} \phi \underset{\zeta \rightarrow 0}{=} \frac{1}{2} \sum_{s=0}^{m} \tilde{\alpha}_{m-s,+} \zeta^{-s}+\tilde{g}_{m,-}+O(\zeta), \quad P \rightarrow P_{\infty-}, \quad \zeta=\lambda^{-1}
$$

Insertion of (5.19) into (5.2) then proves (5.11) for $n=n_{0}$ as $P \rightarrow P_{\infty}$. 
As $P \rightarrow P_{\infty+}$, we need one additional term in the asymptotic expansion of $\widehat{F}_{\underline{m}}$, that is, we will use

$$
\widetilde{F}_{\underline{m}} \underset{\zeta \rightarrow 0}{=} \sum_{s=0}^{m} \widetilde{\alpha}_{m-s,+} \widehat{F}_{s,+}+\tilde{f}_{m-1,-} \zeta+O\left(\zeta^{2}\right)
$$

Using (2.29) yields

$$
\begin{aligned}
\widetilde{G}_{\underline{m}}+\widetilde{F}_{\underline{m}} \phi \underset{\zeta \rightarrow 0}{=}-\frac{1}{2} \sum_{s=0}^{m} \tilde{\alpha}_{m-s,+} \zeta^{-s}+\tilde{g}_{m,-}+\frac{1}{\varepsilon u}\left(\tilde{f}_{m+1,+}-\tilde{f}_{m-1,-}\right)+O(\zeta) \\
\underset{\zeta \rightarrow 0}{=}-\frac{1}{2} \sum_{s=0}^{m} \widetilde{\alpha}_{m-s,+} \zeta^{-s}+\frac{u_{\underline{m}}}{u}+\tilde{g}_{m,+}+O(\zeta), \quad P \rightarrow P_{\infty+}, \quad \zeta=\lambda^{-1}
\end{aligned}
$$

Insertion of (5.22) into (5.2) then proves (5.11) for $n=n_{0}$ as $P \rightarrow P_{\infty+}$. Using Theorem 4.1 again, one obtains in the same manner as $P \rightarrow P_{0, \pm}$,

$$
\widehat{G}_{s,-}+\widehat{F}_{s,-} \phi(P) \underset{\zeta \rightarrow 0}{=} \pm \frac{1}{2} \zeta^{-s}+\frac{\hat{g}_{0,-} \mp \frac{1}{2}}{\hat{f}_{0,-}} \hat{f}_{s,-}+O(\zeta), \quad P \rightarrow P_{0, \pm}, \quad \zeta=\lambda
$$

Since

$$
\widetilde{G}_{\underline{m}}=\sum_{\zeta \rightarrow 0}^{m} \tilde{\alpha}_{m=0} \widehat{G}_{s,-}+\tilde{g}_{m,+}+O(\zeta), \quad \widetilde{F}_{\underline{m}} \underset{\zeta \rightarrow 0}{=} \sum_{s=0}^{m} \tilde{\alpha}_{m-s,+} \widehat{F}_{s,-}+\tilde{f}_{m,+}+O(\zeta),
$$

Using (2.29) and (3.6), as $P \rightarrow P_{0, \pm}, \zeta=\lambda$, one obtains

$$
\begin{gathered}
\widetilde{G}_{\underline{m}}+\widetilde{F}_{\underline{m}} \phi \underset{\zeta \rightarrow 0}{=} \pm \frac{1}{2} \sum_{s=0}^{m} \tilde{\alpha}_{m-s,+} \zeta^{-s}+\tilde{g}_{m,+}-\frac{\hat{g}_{0,-} \mp \frac{1}{2}}{\hat{f}_{0,-}}\left(\tilde{f}_{m,+}-\tilde{f}_{m,-}\right)+O(\zeta) \\
\underset{\zeta \rightarrow 0}{=}\left\{\begin{array}{l}
\frac{1}{2} \sum_{s=0}^{m} \tilde{\alpha}_{m-s,+} \zeta^{-s}+\frac{v_{t_{m}}^{-}}{v^{-}}+\tilde{g}_{m,-}+O(\zeta), \quad \text { as } P \rightarrow P_{0,+}, \quad \zeta=\lambda, \\
-\frac{1}{2} \sum_{s=0}^{m} \tilde{\alpha}_{m-s,+} \zeta^{-s}+\tilde{g}_{m,+}+O(\zeta), \quad \text { as } P \rightarrow P_{0,-}, \quad \zeta=\lambda
\end{array}\right.
\end{gathered}
$$

Insertion of (5.25) into (5.2) then proves (5.12) for $n=n_{0}$ as $P \rightarrow P_{0, \pm}$. Hence, according to the definition of $\psi_{1}$ in (5.2) and (5.4), we can arrive at (5.11) and (5.12).

Next, we shall derive the representations of $\phi, \psi_{1}$ and $u\left(n, t_{\underline{m}}\right), v\left(n, t_{\underline{m}}\right)$ in terms of the Riemann theta functions. Let $\omega_{P_{+}, P_{-}}^{(3)}$ be the normal differential of the third kind holomorphic on $\mathscr{K}_{N} \backslash\left\{P_{+}, P_{-}\right\}$ with simple poles at $P_{+}$and $P_{-}$, corresponding residues 1 and -1 , respectively. In particular, one obtains for $\omega_{P_{0,-}, P_{\infty \pm}}^{(3)}, \quad \omega_{P_{0,+}, P_{\infty+}}^{(3)}$

$$
\begin{array}{ll}
\omega_{P_{0,-}, P_{\infty \pm}}^{(3)}=\frac{y+y_{0,-}}{2 \lambda} \frac{d \lambda}{y} \mp \frac{1}{2 y} \prod_{j=1}^{N}\left(\lambda-\beta_{ \pm, j}\right) d \lambda, & P_{0,-}=\left(0, y_{0,-}\right), \\
\omega_{P_{0,+}, P_{\infty+}}^{(3)}=\frac{y+y_{0,+}}{2 \lambda} \frac{d \lambda}{y}-\frac{1}{2 y} \prod_{j=1}^{N}\left(\lambda-\beta_{1, j}\right) d \lambda, & P_{0,+}=\left(0, y_{0,+}\right),
\end{array}
$$

where $\beta_{ \pm, j}, \beta_{1, j} \in \mathbb{C}, j=1, \ldots, N$, are constants that are uniquely determined by the requirement of vanishing $a$-periods. The explicit formula (5.26) then implies the following asymptotic expansions 
( using the local coordinate $\zeta=\lambda^{-1}$ near $P_{\infty \pm}$ and $\zeta=\lambda$ near $P_{0, \pm}$ )

$$
\begin{gathered}
\int_{P_{0}}^{P} \omega_{P_{0,-}, P_{\infty+}}^{(3)}=\left\{\begin{array}{c}
-\ln (\zeta) \\
0
\end{array}\right\}+\omega_{0}^{\infty \pm}\left(P_{0,-}, P_{\infty+}\right)+O(\zeta) \text { as } \quad P \rightarrow P_{\infty, \pm}, \\
\int_{P_{0}}^{P} \omega_{P_{0,-}, P_{\infty+}}^{(3)}=\left\{\begin{array}{c}
0 \\
\ln (\zeta)
\end{array}\right\}+\omega_{0}^{0 \pm}\left(P_{0,-}, P_{\infty+}\right)+O(\zeta) \text { as } \quad P \rightarrow P_{0, \pm}, \\
\int_{P_{0}}^{P} \omega_{P_{0,-}, P_{\infty-}}^{(3)}=\left\{\begin{array}{c}
0 \\
\zeta \rightarrow 0 \\
-\ln (\zeta)
\end{array}\right\}+\omega_{0}^{\infty \pm}\left(P_{0,-}, P_{\infty-}\right)+O(\zeta) \text { as } \quad P \rightarrow P_{\infty, \pm}, \\
\int_{P_{0}}^{P} \omega_{P_{0,-}, P_{\infty-}}^{(3)}=\left\{\begin{array}{c}
\zeta \rightarrow 0 \\
0 \\
\ln (\zeta)
\end{array}\right\}+\omega_{0}^{0 \pm}\left(P_{0,-}, P_{\infty-}\right)+O(\zeta) \text { as } \quad P \rightarrow P_{0, \pm}, \\
\int_{P_{0}}^{P} \omega_{P_{0,+}, P_{\infty+}}^{(3)}=\left\{\begin{array}{c}
-\ln (\zeta) \\
0
\end{array}\right\}+\omega_{0}^{0 \pm}\left(P_{0,+}, P_{\infty+}\right)+O(\zeta) \text { as } \quad P \rightarrow P_{\infty \pm}, \\
\int_{P_{0}}^{P} \omega_{P_{0,+}, P_{\infty+}}^{(3)}=\left\{\begin{array}{c}
\zeta \rightarrow 0 \\
\ln (\zeta) \\
0
\end{array}\right\}+\omega_{0}^{0 \pm}\left(P_{0,+}, P_{\infty+}\right)+O(\zeta) \text { as } \quad P \rightarrow P_{0, \pm} .
\end{gathered}
$$

Let $\omega_{P_{\infty \pm}, q}^{(2)}$ and $\omega_{P_{0, \pm}, q}^{(2)}$ be the normalized differentials of the second kind holomorphic with a unique pole at $P_{\infty \pm}$ and $P_{0, \pm}$, respectively, and principal parts

$$
\begin{array}{lll}
\omega_{P_{\infty \pm}, q}^{(2)} \underset{\zeta \rightarrow 0}{=}\left(\zeta^{-2-q}+O(1)\right) d \zeta, & P \rightarrow P_{\infty, \pm}, \quad \zeta=\lambda^{-1}, & q \in \mathbb{N}_{0}, \\
\omega_{P_{0, \pm}, q}^{(2)} \underset{\zeta \rightarrow 0}{=}\left(\zeta^{-2-q}+O(1)\right) d \zeta, & P \rightarrow P_{0, \pm}, \quad \zeta=\lambda, \quad q \in \mathbb{N}_{0} .
\end{array}
$$

with vanishing a-periods,

$$
\int_{a_{j}} \omega_{P_{\infty \pm, q}}^{(2)}=\int_{a_{j}} \omega_{P_{0, \pm, q}}^{(2)}=0, \quad j=1, \ldots, N
$$

Moreover, we define

$$
\widetilde{\Omega}_{\underline{m}}^{(2)}=\frac{1}{2}\left(\sum_{s=1}^{m} s \tilde{\alpha}_{m-s,+}\left(\omega_{P_{0,+, s-1}}^{(2)}-\omega_{P_{0,-, s-1}}^{(2)}\right)-\sum_{s=1}^{m} s \tilde{\alpha}_{m-s,+}\left(\omega_{P_{\infty+, s-1}}^{(2)}-\omega_{P_{\infty, s-1}}^{(2)}\right)\right),
$$

where $\tilde{\alpha}_{l, \pm}$, are the summation constants in $\widetilde{F}_{\underline{m}}$. The corresponding vector of b-periods of $\widetilde{\Omega}_{\underline{m}}^{(2)} /(2 \pi i)$ is then denoted by

$$
\underline{U_{m}}(2)=\left(\widetilde{U}_{\underline{m}, 1}^{(2)}, \cdots, \widetilde{U}_{\underline{m}, N}^{(2)}\right), \quad \widetilde{U}_{\underline{m}, j}^{(2)}=\frac{1}{2 \pi i} \int_{b_{j}} \widetilde{\Omega}_{\underline{m}}^{(2)}, \quad j=1, \cdots, N .
$$


Finally, we abbreviate

$$
\widetilde{\Omega}_{\underline{m}}^{\infty \pm}=\lim _{P \rightarrow P_{\infty \pm}}\left(\int_{Q_{0}}^{P} \widetilde{\Omega}_{\underline{m}}^{(2)} \mp \frac{1}{2} \sum_{s=0}^{m} \tilde{\alpha}_{m-s,+} \zeta^{-s}\right), \quad \widetilde{\Omega}_{\underline{m}}^{0, \pm}=\lim _{P \rightarrow P_{0, \pm}}\left(\int_{P_{0}}^{P} \widetilde{\Omega}_{\underline{m}}^{(2)} \pm \frac{1}{2} \sum_{s=0}^{m-1} \tilde{\alpha}_{m-s,+} \zeta^{-s}\right) .
$$

If $D_{\hat{\underline{\mu}}\left(n, t_{\underline{m}}\right)}, D_{\hat{\underline{\hat{v}}}\left(n, t_{\underline{\underline{m}}}\right)}$ are assumed to be nonspecial [3], then according to the Riemann's vanishing theorem $[3,12,14]$, the definition and asymptotic properties of the meromorphic function $\phi\left(P, n, t_{\underline{m}}\right), \phi$ has the expression of the following type:

$$
\phi\left(P, n, t_{\underline{m}}\right)=N\left(n, t_{\underline{m}}\right) \frac{\theta\left(P, D_{\underline{\hat{\hat{v}}}\left(n, t_{\underline{\underline{t}}}\right)}\right)}{\theta\left(P, D_{\underline{\hat{\mu}}\left(n, \underline{t}_{\underline{m}}\right)}\right)} \exp \left(\int_{P_{0}}^{P} \omega_{P_{0,-}, P_{\infty+}}^{(3)}\right),
$$

where $N\left(n, t_{\underline{m}}\right)$ is independent of $P \in \mathscr{K}_{N}$.

Given these preparations, the theta function representations for $\phi\left(P, n, t_{\underline{m}}\right), \psi_{1}\left(P, n, n_{0}, t_{\underline{m}}, t_{0, \underline{m}}\right)$, $u\left(n, t_{\underline{m}}\right), v\left(n, t_{\underline{m}}\right)$ then read as follows

Theorem 5.3 Assume Hypothesis 2.1 and suppose that (2.29) and (3.2) hold. In addition, let $P=(\lambda, y) \in \mathscr{K}_{N} \backslash\left\{P_{\infty+}, P_{\infty-}, P_{0,+}, P_{0,-}\right\}$ and $\left(n, n_{0}, t_{\underline{m}}, t_{0, \underline{m}}\right) \in \mathbb{Z}^{2} \times \mathbb{R}^{2}$, and for each $\left(n, t_{\underline{m}}\right) \in \mathbb{Z} \times \mathbb{R}$, $D_{\underline{\hat{\mu}}\left(n, t_{\underline{m}}\right)}, \quad D_{\underline{\hat{v}}\left(n, t_{\underline{m}}\right)}$ is nonspecial. Then,

$$
\begin{gathered}
\phi\left(P, n, t_{\underline{m}}\right)=N\left(n, t_{\underline{m}}\right) \frac{\theta\left(P, D_{\underline{\hat{v}}\left(n, t_{\underline{m}}\right)}\right)}{\theta\left(P, D_{\underline{\hat{\mu}}\left(n, t_{\underline{m}}\right)}\right)} \exp \left(\int_{P_{0}}^{P} \omega_{P_{0,-}, P_{\infty+}}^{(3)}\right), \\
\psi_{1}\left(P, n, n_{0}, t_{\underline{m}}, t_{0, \underline{m}}\right)=N\left(n, n_{0}, t_{\underline{m}}, t_{0, \underline{m}}\right) \frac{\theta\left(P, D_{\hat{\mu}}\left(n, t_{\underline{m}}\right)\right.}{\theta\left(P, D_{\hat{\mu}\left(n_{0}, t_{0, \underline{m}}\right)}\right)} \\
\times \exp \left(\left(n-n_{0}\right) \int_{P_{0}}^{P} \omega_{P_{0,-}, P_{\infty-}}^{(3)}-\left(n-n_{0}\right) \int_{P_{0}}^{P} \omega_{P_{0,+}, P_{\infty+}}^{(3)}-\left(t_{\underline{m}}-t_{0, \underline{m}}\right) \int_{P_{0}}^{P} \widetilde{\Omega}_{\underline{m}}^{(2)}\right),
\end{gathered}
$$

where

$$
\begin{aligned}
& N\left(n, t_{\underline{m}}\right)=\exp \left[-\frac{1}{2}\left(\omega_{0}^{\infty+}\left(P_{0,-}, P_{\infty+}\right)+\omega_{0}^{0-}\left(P_{0,-}, P_{\infty+}\right)\right)\right] \\
& \times\left(\varepsilon \frac{\theta\left(P_{\infty+}, D_{\hat{\hat{\mu}}\left(n, t_{\underline{\underline{m}}}\right)}\right) \theta\left(P_{0,-}, D_{\hat{\underline{\mu}}\left(n, t_{\underline{\underline{m}}}\right)}\right)}{\theta\left(P_{\infty+}, D_{\underline{\hat{v}}\left(n, t_{\underline{m}}\right)}\right) \theta\left(P_{0,-}, D_{\underline{\hat{v}}\left(n, t_{\underline{m}}\right)}\right)}\right)^{\frac{1}{2}}, \\
& N\left(n, n_{0}, t_{\underline{m}}, t_{0, \underline{m}}\right)=\exp \left[\left(n-n_{0}\right)\left(\omega_{0}^{\infty-}\left(P_{0,+}, P_{\infty+}\right)-\omega_{0}^{\infty-}\left(P_{0,-}, P_{\infty-}\right)\right)+\left(t_{\underline{m}}-t_{0, \underline{m}}\right) \widetilde{\Omega}_{\underline{m}}^{\infty-}\right] \\
& \times \exp \left(\int_{t_{0, \underline{m}}}^{t_{\underline{\underline{m}}}} \tilde{g}_{m,-}\left(n_{0}, s\right) d s\right) \frac{\theta\left(P_{\infty-}, D_{\underline{\hat{\mu}}\left(n_{0}, t_{0, \underline{m}}\right)}\right)}{\theta\left(P_{\infty-}, D_{\underline{\hat{\mu}}\left(n, \underline{t}_{\underline{m}}\right)}\right)} .
\end{aligned}
$$

The Abel map linearizes the auxiliary divisors $D_{\hat{\mu}\left(n, t_{\underline{\underline{m}}}\right)}, D_{\hat{v}\left(n, \underline{\underline{m}}_{\underline{\underline{m}}}\right)}$ in the sense that $\underline{\rho}^{(1)}\left(n, t_{\underline{m}}\right)=\underline{\rho}^{(1)}\left(n_{0}, t_{0, \underline{m}}\right)+\left(\mathscr{A}\left(P_{0,+}\right)-\mathscr{A}\left(P_{0,-}\right)+\mathscr{A}\left(P_{\infty-}\right)-\mathscr{A}\left(P_{\infty+}\right)\right)\left(n-n_{0}\right)+\underline{Y}^{(\underline{m})}\left(t_{\underline{m}}-t_{0, \underline{m}}\right)$,
$\underline{\rho}^{(2)}\left(n, \underline{t}_{\underline{m}}\right)=\underline{\rho}^{(2)}\left(n_{0}, t_{0, \underline{\underline{m}}}\right)+\left(\mathscr{A}\left(P_{0,+}\right)-\mathscr{A}\left(P_{0,-}\right)+\mathscr{A}\left(P_{\infty-}\right)-\mathscr{A}\left(P_{\infty+}\right)\right)\left(n-n_{0}\right)-\underline{Y}^{(\underline{m})}\left(\underline{t}_{\underline{m}}-t_{0, \underline{m}}\right)$. 
Finally, $u\left(n, t_{\underline{m}}\right), v\left(n, t_{\underline{m}}\right)$ are of the form

$$
\begin{aligned}
& u\left(n, t_{\underline{\underline{m}}}\right)=-\exp \left[\frac{1}{2}\left(\omega_{0}^{0-}\left(P_{0,-}, P_{\infty+}\right)-\omega_{0}^{\infty+}\left(P_{0,-}, P_{\infty+}\right)\right)\right]\left(\varepsilon \frac{\theta\left(P_{\infty,+}, D_{\hat{\hat{\mu}}\left(n, t_{\underline{\underline{m}}}\right)}\right) \theta\left(P_{0,-}, D_{\underline{\hat{v}}\left(n, t_{\underline{\underline{m}}}\right)}\right)}{\theta\left(P_{\infty,+}, D_{\underline{\hat{\hat{\hat{y}}}}\left(n, t_{\underline{\underline{t}}}\right)}\right) \theta\left(P_{0,+}, D_{\underline{\hat{\mu}}\left(n, t_{\underline{\underline{m}}}\right)}\right)}\right)^{\frac{1}{2}}, \\
& v\left(n, t_{\underline{m}}\right)=v\left(n_{0}, t_{0, \underline{m}}\right) \exp \left[( n - n _ { 0 } ) \left(\omega_{0}^{\infty-}\left(P_{0,+}, P_{\infty+}\right)-\omega_{0}^{\infty-}\left(P_{0,-}, P_{\infty-}\right)+\omega_{0}^{0,+}\left(P_{0,-}, P_{\infty-}\right)\right.\right. \\
& \left.\left.-\omega_{0}^{0,+}\left(P_{0,+}, P_{\infty++}\right)\right)+\left(t_{\underline{m}}-t_{0, \underline{m}}\right)\left(\widetilde{\Omega}_{\underline{m}}^{\infty-}-\widetilde{\Omega}_{\underline{m}}^{0,+}\right)\right] \\
& \times \frac{\theta\left(P_{0,+}, D_{\underline{\hat{\mu}}^{+}\left(n, t_{\underline{m}}\right)}\right) \theta\left(P_{\infty-}, D_{\underline{\hat{\mu}}^{+}\left(n_{0}, t_{0, \underline{m}}\right)}\right)}{\theta\left(P_{0,+}, D_{\underline{\hat{\mu}}^{+}\left(n_{0}, t_{0, \underline{m}}\right)}\right) \theta\left(P_{\infty-}, D_{\underline{\hat{\mu}}^{+}\left(n, t_{\underline{m}}\right.}\right)} .
\end{aligned}
$$

Proof: Applying Abel's theorem to (5.14), we have

$$
\underline{\rho}^{(1)}\left(n, t_{\underline{m}}\right)=\underline{\rho}^{(1)}\left(n_{0}, t_{\underline{m}}\right)-\left(\mathscr{A}\left(P_{0,+}\right)-\mathscr{A}\left(P_{0,-}\right)+\mathscr{A}\left(P_{\infty-}\right)-\mathscr{A}\left(P_{\infty+}\right)\right)\left(n-n_{0}\right),
$$

on the other hand, according to theorem $4.5\left(n=n_{0}\right)$,

$$
\begin{aligned}
& \underline{\rho}^{(1)}\left(n_{0}, t_{\underline{m}}\right)=\underline{\rho}^{(1)}\left(n_{0}, t_{0, \underline{m}}\right)+\underline{Y}^{(\underline{m})}\left(t_{\underline{m}}-t_{0, \underline{m}}\right), \\
& \underline{\rho}^{(2)}\left(n_{0}, t_{\underline{m}}\right)=\underline{\rho}^{(2)}\left(n_{0}, t_{0, \underline{m}}\right)-\underline{Y}^{(\underline{m})}\left(t_{\underline{m}}-t_{0, \underline{m}}\right),
\end{aligned}
$$

thus the first expression of (5.41) holds. According to the divisor of $\phi\left(\cdot n, t_{m}\right)$ we can obtain the linear equivalence $D_{P_{0,-} \underline{\hat{v}}\left(n, t_{m}\right)} \sim D_{P_{\infty}+\underline{\hat{\mu}}\left(n, t_{m}\right)}$, that is,

$$
\underline{\rho}^{(2)}\left(n, t_{m}\right)+\mathscr{A}\left(P_{0,-}\right)=\underline{\rho}^{(1)}\left(n, t_{m}\right)+\mathscr{A}\left(P_{\infty+}\right),
$$

then we can prove the second expression of (5.41).

By equation (5.1), and the Riemann's vanishing theorem, $\phi\left(P, n, \underline{t_{m}}\right) \exp \left(-\int_{P_{0}}^{P} \omega_{P_{0,-}, P_{\infty+}}^{(3)}\right)$ must be of the type

$$
\phi\left(P, n, t_{\underline{m}}\right) \exp \left(-\int_{P_{0}}^{P} \omega_{P_{0,-}, P_{\infty+}}^{(3)}\right)=N\left(n, t_{\underline{m}}\right) \frac{\theta\left(P, D_{\underline{\hat{v}}\left(n, t_{\underline{m}}\right)}\right)}{\theta\left(P, D_{\underline{\hat{\mu}}\left(n, t_{\underline{m}}\right)}\right)}
$$

for some constant $N\left(n, t_{\underline{m}}\right)$. First, if the local coordinate $\zeta=\lambda^{-1}$ is introduced near $P_{\infty \pm}$, we can conclude from the definition (4.5) of the normalized basis $\omega_{j}$ that

$$
\begin{aligned}
\underline{\omega} & =\left(\omega_{1}, \ldots, \omega_{N}\right)=\mp \sum_{l=1}^{N} \frac{\underline{C}_{l} \lambda^{l-1} d \lambda}{\prod_{j=1}^{2 N+2}\left(\lambda-\lambda_{j}\right)^{\frac{1}{2}}} \\
& = \pm \sum_{l=1}^{N} \frac{\underline{C}_{l} \zeta^{N-j} d \zeta}{\prod_{j=1}^{2 N+2}\left(1-\lambda_{j} \zeta^{2}\right)^{\frac{1}{2}}} \\
& = \pm\left(\underline{C}_{N}+O(\zeta)\right) d \zeta, \quad \text { as } P \rightarrow P_{\infty \pm} .
\end{aligned}
$$


Hence, the Abel map

$$
\begin{aligned}
\mathscr{A}(P) & =\int_{P_{0}}^{P} \underline{\omega}=\int_{P_{0}}^{P_{\infty \pm}} \underline{\omega}+\int_{P_{\infty \pm}}^{P} \underline{\omega} \\
& =\mathscr{A}\left(P_{\infty \pm}\right)+\int_{P_{\infty \pm}}^{P} \underline{\omega} \\
& =\mathscr{A}\left(P_{\infty \pm}\right) \pm \underline{C}_{N} \zeta+O\left(\zeta^{2}\right) .
\end{aligned}
$$

Therefore, expanding the ratios of Riemann theta functions in (5.37), we obtain

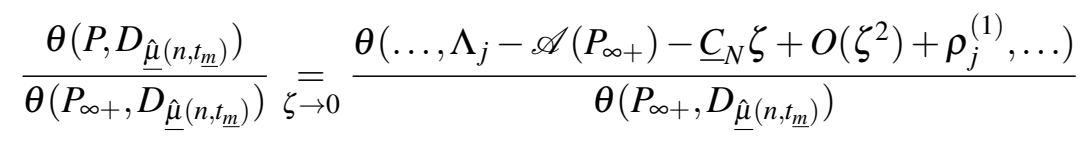

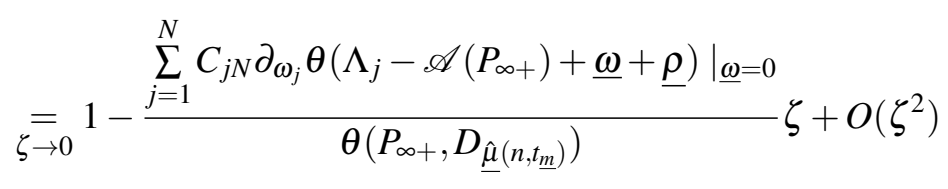

$$
\begin{aligned}
& \underset{\zeta \rightarrow 0}{=} 1-\sum_{j=1}^{N} C_{j N} \partial_{\omega_{j}} \ln \theta\left(P_{\infty+}, D_{\underline{\hat{\mu}}\left(n, t_{\underline{m}}\right)}\right) \zeta+O\left(\zeta^{2}\right) \quad \text { as } P \rightarrow P_{\infty+},
\end{aligned}
$$

and the same formula for the theta function ratio involving $D_{\underline{\hat{\hat{v}}}\left(n, t_{m}\right)}$, that is

$$
\frac{\theta\left(P, D_{\underline{\hat{v}}\left(n, t_{\underline{\underline{m}}}\right)}\right)}{\theta\left(P_{\infty+}, D_{\underline{\hat{v}}\left(n, t_{\underline{\underline{m}}}\right)}\right)} \underset{\zeta \rightarrow 0}{=} 1-\sum_{j=1}^{N} C_{j N} \partial_{\omega_{j}} \ln \theta\left(P_{\infty+}, D_{\underline{\hat{v}}\left(n, t_{\underline{\underline{m}}}\right)}\right) \zeta+O\left(\zeta^{2}\right) \quad \text { as } P \rightarrow P_{\infty+} .
$$

Finally, from the representation (5.37) of $\phi$, we get

$$
\phi \underset{\zeta \rightarrow 0}{=} N\left(n, t_{\underline{m}}\right) \frac{\theta\left(P_{\infty+}, D_{\hat{\hat{v}}\left(n, t_{\underline{\underline{m}}}\right)}\right)}{\theta\left(P_{\infty+}, D_{\underline{\hat{\mu}}\left(n, t_{\underline{m}}\right)}\right)} \exp \left(\omega_{0}^{\infty+}\left(P_{0,-}, P_{\infty+}\right)\right) \zeta^{-1}+O(1) \quad \text { as } P \rightarrow P_{\infty+},
$$

which together with the first expression of (5.5) and (5.27) shows that

$$
-\frac{1}{\varepsilon u\left(n, t_{\underline{m}}\right)}=N\left(n, t_{\underline{m}}\right) \frac{\theta\left(P_{\infty+}, D_{\hat{\hat{\hat{v}}}\left(n, t_{\underline{m}}\right)}\right)}{\theta\left(P_{\infty+}, D_{\underline{\hat{\mu}}\left(n, t_{\underline{m}}\right)}\right)} \exp \left(\omega_{0}^{\infty+}\left(P_{0,-}, P_{\infty+}\right)\right) .
$$

If we introduce the local coordinate $\zeta=\lambda$ near $P_{0, \pm}$, in a similar way, from the representation (5.37) of $\phi$, we get

$$
\phi \underset{\zeta \rightarrow 0}{=} N\left(n, t_{\underline{m}}\right) \frac{\theta\left(P_{0,-}, D_{\underline{\hat{\hat{v}}}\left(n, t_{\underline{\underline{m}}}\right)}\right)}{\theta\left(P_{0,-}, D_{\underline{\hat{\mu}}\left(n, t_{\underline{m}}\right)}\right)} \exp \left(\omega_{0}^{0,-}\left(P_{0,-}, P_{\infty+}\right)\right) \zeta+O\left(\zeta^{2}\right) \quad \text { as } P \rightarrow P_{0,-},
$$

together with the second expression of (5.6), which shows that

$$
-u\left(n, t_{\underline{m}}\right)=N\left(n, t_{\underline{m}}\right) \frac{\theta\left(P_{0,-}, D_{\underline{\hat{\hat{v}}}\left(n, t_{\underline{m}}\right)}\right)}{\theta\left(P_{0,-}, D_{\underline{\hat{\mu}}\left(n, \underline{t}_{\underline{m}}\right)}\right)} \exp \left(\omega_{0}^{0,-}\left(P_{0,-}, P_{\infty+}\right)\right) .
$$

Combining (5.49) and (5.51), we can obtain the expression (5.39) for $N\left(n, t_{\underline{m}}\right)$. Inserting (5.39) into (5.51) yields the Riemann theta function expression (5.42) for $u\left(n, t_{m}\right)$.

To prove (5.38), we denote its right-hand side by $\widetilde{\psi_{1}}$. From the definition (5.2) of $\psi_{1}\left(P, n, n_{0}, t_{\underline{m}}, t_{0, \underline{m}}\right)$ and $(5.14)$, one can conclude that it has simple zeros at $P_{0,-}, P_{\infty+}$ and $\hat{\mu}_{k}\left(n, t_{\underline{m}}\right)$, 
$k=1, \ldots, N$, and simple poles at $P_{0,+}, P_{\infty-}$ and $\hat{\mu}_{k}\left(n_{0}, t_{0, \underline{m}}\right), k=1, \ldots, N$. On the other hand, by (5.29)-(5.32) and the expression of $\widetilde{\psi_{1}}$, we know that $\widetilde{\psi_{1}}$ and $\psi_{1}$ shares the same local behavior and identical essential singularities at $P_{\infty \pm}$ and $P_{0, \pm}$. Then, according to the Riemann-Roch uniqueness theorem, $\psi_{1}\left(P, n, n_{0}, t_{\underline{m}}, t_{0, \underline{m}}\right)$ and $\widetilde{\psi_{1}}$ coincide up to a multiple constant, that is, $\psi_{1}\left(P, n, n_{0}, t_{\underline{m}}, t_{0, \underline{m}}\right)$ must be of the form (5.33) for some constant $N\left(n, n_{0}, t_{\underline{m}}, t_{0, \underline{\underline{m}}}\right)$ to be determined. To determine the constant $N\left(n, n_{0}, t_{\underline{m}}, t_{0, \underline{m}}\right)$, one compares the asymptotic expansions of $\psi_{1}\left(P, n, n_{0}, t_{\underline{m}}, t_{0, \underline{m}}\right)$ for $P \rightarrow P_{\infty-}$ in (5.11) and (5.38), which yields (5.40). Remaining to be computed is the expression for $v\left(n, t_{\underline{m}}\right)$. Comparing the asymptotic expression of $\psi_{1}\left(n, n_{0}, t_{\underline{m}}, t_{0, \underline{m}}\right)$ for $P \rightarrow P_{\infty,+}$ in (5.11)and (5.38) shows

$$
\begin{aligned}
& \frac{v^{-}\left(n, t_{\underline{m}}\right)}{v^{-}\left(n_{0}, t_{0, \underline{m}}\right)} \exp \left(\int_{t_{0, m}}^{t_{\underline{m}}} \tilde{g}_{m,-}\left(n_{0}, s\right) d s\right) \\
= & N\left(n, n_{0}, t_{\underline{m}}, t_{0, \underline{m}}\right) \exp \left[\left(n-n_{0}\right)\left(\omega_{0}^{0,+}\left(P_{0,-}, P_{\infty-}\right)-\omega_{0}^{0,+}\left(P_{0,+}, P_{\infty+}\right)\right)-\left(t_{\underline{m}}-t_{0, \underline{m}}\right) \widetilde{\Omega}_{\underline{\underline{m}}}^{0,+}\right] \\
& \times \frac{\theta\left(P_{0,+}, D_{\underline{\hat{\mu}}\left(n, t_{\underline{m}}\right)}\right)}{\theta\left(P_{0,+}, D_{\underline{\hat{\mu}}\left(n_{0}, t_{0, \underline{m}}\right)}\right)}
\end{aligned}
$$

and inserting $N\left(n, n_{0}, t_{\underline{m}}, t_{0, \underline{m}}\right)$ proves (5.43).

\section{Acknowledgments}

This work was supported by the National Natural Science Foundation of China (project nos. 11331008 and 11171312) and Docter Foundation of Henan Institute of Engineering (project no: D2014004).

\section{References}

[1] M. J. Ablowitz and J. F. Ladik, Nonlinear differential-difference equation, J. Math. Phys. 16 (1975) 598-603.

[2] S. Ahmad and A. R. Chowdhury, On the quasi-periodic solutions to the discrete nonlinear Schrödinger equation, J. Math. Phys. 28 (1987) 134-137.

[3] S. Ahmad and A. R. Chowdhury, On the quasi-periodic solutions to the discrete nonlinear Schrödinger equation, J. Phys. A: Math. Gen. 20 (1987) 293-303.

[4] S. J. Alber, On finite-zone solutions of relativistic Toda lattices, Lett. Math. Phys. 17 (1989) 149-155.

[5] N. N. Bogolyubov, A. K . Prikarpatskii, and V. G. Samoilenko, A discrete periodic problem for the modified nonlinear Korteweg-de Vries equation, Theoret. and Math. Phys. 50 (1982) 75-81.

[6] N. N. Bogolyubov and A. K. Prikarpatskii, The inverse periodic problem for a discrete approximation of nonlinear Schrödinger equation, Sov. Phys. Dokl. 27 (1982) 113-116.

[7] N. N. Bogolyubov, A. K . Prikarpatskii, and V. G. Samoilenko, A discrete periodic problem for the modified nonlinear Korteweg-de Vries equation, Theoret. and Math. Phys. 50 (1982) 75-81.

[8] W. Bulla, F. Gesztesy, H. Holden and G. Teschl, Algebro-geometric quasi-periodic finite-gap solutions of the Toda and Kac-van Moerbeke hierarchies. Mem. Amer. Math. Soc. 135 (1998) 1-79

[9] C. W. Cao, X. G. Geng and Y. T. Wu, From the special 2+1 Toda lattice to the Kadomtsev-Petviashvili equation, J.Phys. A. Math. Gen. 32 (1999) 8059-8078.

[10] C. W. Cao, X. G. Geng and H. Y. Wang, Algebro-geometric solution of the $2+1$ dimensional Burgers equation with a discrete variable, J. Math. Phys. 43 (2002) 621-643.

[11] H. H. Dai and X. G. Geng, Explicit solutions of the 2+1-dimensional modified Toda lattice through straightening out of the relativistic Toda flows J. Phys. Soc. Jpn . 72 (2003) 3063-3069.

[12] X. G. Geng, H. H. Dai and C. W. Cao, Algebro-geometric constructions of the discrete Ablowitz-Ladik flows and applications, J. Math. Phys. 44 (2003) 4573-4588. 
[13] X. G. Geng, H. H. Dai and J. Y. Zhu, Decomposition of the discrete Ablowitz-Ladik hierarchy, Stud. Appl. Math. 118 (2007) 281-312

[14] J. S. Geronimo, F. Gesztesy, and H. Holden, Algebro-geometric solutions of the Baxter-Szegö difference equation, Comm. Math. Phys. 258 (2005) 149-177.

[15] F. Gesztesy, H. Holden, J. Michor, and G. Teschl, Soliton equations and their algebro-geometric solutions, Volume II: (1+1)-dimensional discrete models (Cambridge University Press, Cambridge, 2008).

[16] U. Göktas and W. Hereman, Computation of conservation laws for nonlinear lattices, Physica D. 123 (1998) 425-426.

[17] P. Griffiths and J. Harris, Principles of algebraic geometry (Wiley, New York, 1994).

[18] R. Hirota, Exact N-soliton solution of a nonlinear lumped networke quation, J. Phys. Soc. Jpn. 35 (1973) 289-294.

[19] D. Mumford, Tata lectures on theta II ( Birkhäuser, Boston, 1984).

[20] K. Narita, Solutions for a system of difference-differential equations related to the self-dual network equation, Prog. Theor. Phys. 106 (2001) 1079-1096.

[21] A. B. Shabat and R. I. Yamilov, Symmetries of nonlinear lattices, Leningrad Math. J. 2 (1991) 377-400.

[22] M. Toda, Theroy of Nonlinear Lattice (Springer, berlin, 1981).

[23] M. Wadati, Transformation Theories for Nonlinear Discrete Systems, Prog. Theor. Phys. Suppl. 59 (1976) 36-63.

[24] J. Zhou, D. J. Zhang and S.L. Zhao, Breathers and limit solutions of the nonlinear lumped self-dual network equation, Phys. Lett. A, 373 (2009) 3248-3258. 